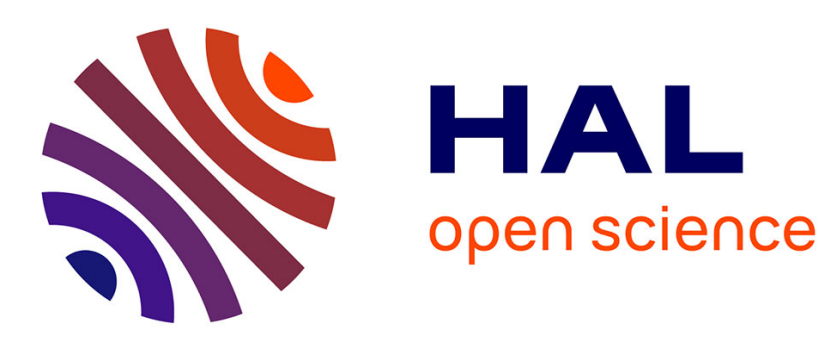

\title{
A 3D DISCRETE DUALITY FINITE VOLUME METHOD FOR NONLINEAR ELLIPTIC EQUATIONS
}

Yves Coudière, Florence Hubert

\section{To cite this version:}

Yves Coudière, Florence Hubert. A 3D DISCRETE DUALITY FINITE VOLUME METHOD FOR NONLINEAR ELLIPTIC EQUATIONS. SIAM Journal on Scientific Computing, 2011, 33 (4), pp.1739-1764. 10.1137/100786046 . hal-00456837

\section{HAL Id: hal-00456837 https://hal.science/hal-00456837}

Submitted on 15 Feb 2010

HAL is a multi-disciplinary open access archive for the deposit and dissemination of scientific research documents, whether they are published or not. The documents may come from teaching and research institutions in France or abroad, or from public or private research centers.
L'archive ouverte pluridisciplinaire HAL, est destinée au dépôt et à la diffusion de documents scientifiques de niveau recherche, publiés ou non, émanant des établissements d'enseignement et de recherche français ou étrangers, des laboratoires publics ou privés. 


\title{
A 3D DISCRETE DUALITY FINITE VOLUME METHOD FOR NONLINEAR ELLIPTIC EQUATIONS*
}

\author{
YVES COUDIÈRE ${ }^{\dagger}$ AND FLORENCE HUBERT H $^{\ddagger}$
}

\begin{abstract}
Discrete Duality Finite Volume (DDFV) schemes have recently been developed in $2 \mathrm{D}$ to approximate nonlinear diffusion problems on general meshes. In this paper, a 3D extension of these schemes is proposed. The construction of this extension is detailed and its main properties are proved: a priori bounds, well-posedness and error estimates. The practical implementation of this scheme is easy. Numerical experiments are presented to illustrate its good behavior.
\end{abstract}

Key words. Finite-volume methods, Error estimates, Leray-Lions operators.

AMS subject classifications. 35J65, 65N15, 74S10

\section{Introduction.}

1.1. Nonlinear elliptic equations. In this paper, we are interested in the study of a finite volume approximation of solutions to the nonlinear diffusion problem:

$$
-\operatorname{div}(\varphi(z, \nabla u(z)))=f(z), \quad \text { in } \Omega, \quad u=g, \text { on } \partial \Omega,
$$

where $\Omega$ is a bounded polyhedral domain in $\mathbb{R}^{3}$. Consider $p \geq 2$ and $p^{\prime}=\frac{p}{p-1}$. The flux $\varphi: \Omega \times \mathbb{R}^{3} \rightarrow \mathbb{R}^{3}$ in equation (1.1) is supposed to be a Caratheodory function which is strictly monotonic with respect to $\xi \in \mathbb{R}^{3}$ :

$$
(\varphi(z, \xi)-\varphi(z, \eta)) \cdot(\xi-\eta)>0 \text {, for all } \xi \neq \eta \text {, for a.e. } z \in \Omega \text {. }
$$

We also assume that there exist $c_{1}, c_{2}>0, b_{1} \in L^{1}(\Omega), b_{2} \in L^{p^{\prime}}(\Omega)$ such that

$$
\begin{gathered}
\varphi(z, \xi) \cdot \xi \geq c_{1}|\xi|^{p}-b_{1}(z), \text { for all } \xi \in \mathbb{R}^{3} \text {, a.e. } z \in \Omega, \\
|\varphi(z, \xi)| \leq c_{2}|\xi|^{p-1}+b_{2}(z), \text { for all } \xi \in \mathbb{R}^{3} \text {, a.e. } z \in \Omega .
\end{gathered}
$$

These assumptions ensure that $u \mapsto-\operatorname{div}(\varphi(\cdot, \nabla u))$ is a Leray-Lions operator, and in particular (see [22])

$$
\text { the mapping } G \in\left(L^{p}(\Omega)\right)^{3} \mapsto \varphi(\cdot, G(\cdot)) \in\left(L^{p^{\prime}}(\Omega)\right)^{3} \quad \text { is continuous. }
$$

Furthermore, Leray and Lions [22] proved that

THEOREM 1.1. Under assumptions (1.2), (1.3) and (1.4), for any source term $f \in W^{-1, p^{\prime}}(\Omega)$ and boundary data $g \in W^{1-1 / p, p}(\partial \Omega)$, the problem (1.1) has a unique solution $u \in W^{1, p}(\Omega)$.

For sake of simplicity, the numerical method is described in details in the more regular context $f \in L^{p^{\prime}}(\Omega)$ and $g$ continuous on $\partial \Omega$. The possible extension to the general case $g \in W^{1-1 / p, p^{\prime}}(\Omega)$ and $f \in W^{-1, p^{\prime}}(\Omega)$ is briefly explained in section 5 . The computation of the final error estimates requires the additional regularity $u \in$

* The authors were partially supported by l'Agence Nationale de la Recherche under grant ANR VFSitCom, BLAN08_2_311650

${ }^{\dagger}$ Laboratoire de Mathématiques Jean Leray, CNRS - Université de Nantes - ÉCN, UMR 6629, France (Yves. Coudiere@univ-nantes.fr).

${ }^{\ddagger}$ Laboratoire d'Analyse, Topologie et Probabilité, CNRS UMR 6632, Université de Provence, Marseille, France (florence.hubert@cmi.univ-mrs.fr). 
$W^{2, p}(\Omega)$. In view of the Sobolev's injections on $\Omega$ in dimension $d=3$ (see [1]), since $p \geq 2>d / 2$ (and then $\frac{1}{p}-\frac{1}{d}<\frac{1}{d}$ ), the following continuous embeddings hold true

$$
W^{2, p}(\Omega) \subset W^{1, q}(\Omega) \subset C^{0}(\bar{\Omega}) \quad \text { if } \frac{1}{p}-\frac{1}{d}<\frac{1}{q}<\frac{1}{d} .
$$

In this case, the boundary data is supposed to be $g=\gamma_{0}(\bar{g})$ where $\bar{g}$ is in $W^{2, p}(\Omega) \subset$ $C^{0}(\bar{\Omega})$.

The scheme could still be analyzed in the general case, and its numerical properties be essentially preserved, although their proofs require much technical work, see for instance [3].

1.2. The discrete duality finite volume approaches in $3 \mathrm{D}$. Given a finite volume mesh, the 2D DDFV method relies on the diamond formula [9] to compute gradients of the unknown $u$ from finite differences in two independent directions, involving values of $u$ at the centers and at the vertices of the control volumes (see $[16,10,3])$. Hence, the DDFV strategy consists in building two finite volume meshes, namely the cell-centered given mesh and a vertex centered one. In $2 \mathrm{D}$, there is a geometric duality relationship such that the interfaces between control volumes of each of these two meshes can be gathered by pairs. These pairs of edges define the so-called diamond cells, on which the gradient vectors are computed. The diamond cells are quadrilateral as shown on figure 1.1(a).

Considering 3D extensions, three different methods have been proposed up to now in the linear case $\varphi(z, \xi)=\mathbf{K}(z) \xi(\mathbf{K}(z)$ a symmetric uniformly elliptic matrix). In these proposals, the additional mesh of control volumes is built around the vertices of the primal mesh (figures 1.1(c) and 1.1(d)) and the diamond cells are as shown on figure 1.1(b). The gradient is approximated by finite differences on the diamond cells and the scheme is obtained by integrating eq. (1.1) on both the primal and dual control volumes. In $[8,23,2,18]$, each diamond cell is composed of a pair of pyramids having

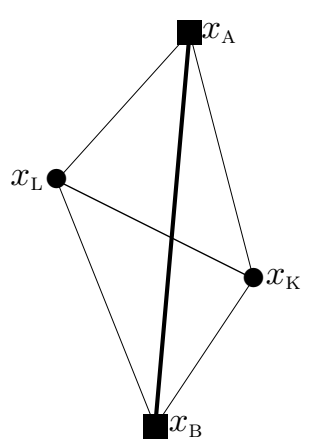

(a) 2D situation

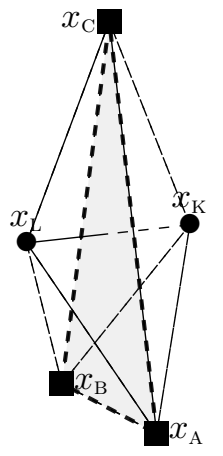

(b) 3D diamond from $[8,2]$.

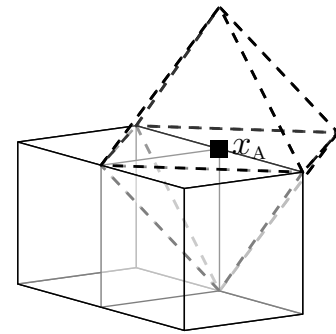

(c) 3D node control volume from $[8,23]$.

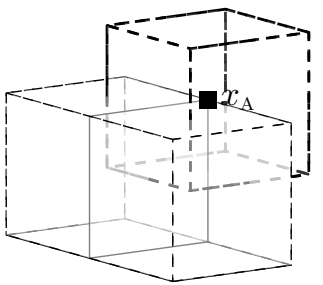

(d) 3D node control volume from $[2,18]$.

FiguRE 1.1. Various schemes.

as base an interface of the primal mesh and vertices the two neighboring centers (see figure 1.1(b)). In $[8,23]$ a $3 \mathrm{D}$ gradient is built from the vertex values of each diamond cell under the condition that the interfaces of the primal mesh are either triangles or quadrangles. This includes locally refined meshes. The construction in $[2,18]$ allows 
more general meshes. The construction of the dual control volumes is specific to each method. Extension to discontinuous permeability has been proposed in [18].

Unlike in the $2 \mathrm{D}$ version, the primal and the dual meshes play a different role: in [23] the domain $\Omega$ is recovered twice by the dual mesh and in [2] the orthogonality condition means that the dual mesh is the Voronoïmesh associated to the vertices of the primal mesh. In reference [17], diamond cells are constructed in a different way:

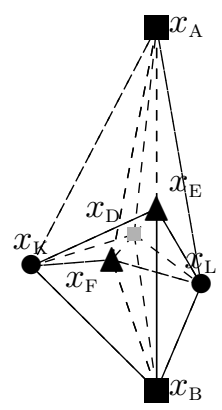

Figure 1.2. View of a diamond cell.

choosing a point in each face of the mesh, the diamond cell is made of two tetrahedral cells that have a common triangular base with vertices the endpoints of one edge of the face and the center of the face; and the two neighboring centers as additional vertices, see figure 1.2. Two auxiliary unknowns, at the centers of the face and of the edge, are introduced to reconstruct the $3 \mathrm{D}$ gradient. With this two additional points, the diamond cell now has 6 vertices, defining 3 independent directions: between the two new points, between the two neighboring centers and between the two endpoints of the edge. It can be constructed a gradient from the 3 finite differences in these directions. But it remains the auxiliary unknowns to eliminate. F. Hermeline suggests several possibilities to eliminate them. The derived schemes are in general non symmetric. Their convergence seems to be difficult to prove.

In any case, three finite differences in independent directions are needed to construct a 3D gradient. The diamond mesh constructed in [17] gives naturally these three independent directions. According to our method, the additional unknowns are computed by integrating the equation on a third family of control volumes associated to the new unknowns at the faces and at the edges of the primal mesh. Like in the $2 \mathrm{D}$ case, the three meshes play a symmetric role, resulting in a scheme that is unexpectedly simple to implement.

Hence, our innovative scheme is based on a three meshes finite volume formulation. The diamond cells have 6 vertices organized in 3 pairs, defining 3 independent directions in $\mathbb{R}^{3}$. The approximate gradient is easily obtained by the 3 corresponding finite differences. The scheme is naturally symmetric. It is derived as a natural generalization of the 2D method, and could eventually be generalized to higher dimensions. Assembling the matrix consists in gathering some local $3 \times 3$ anisotropic Gram matrices (section 5.2).

This paper specifies the construction of this 3D DDFV scheme for a nonlinear elliptic equation, states and proves the main properties of this scheme, including some error estimates, and finally presents several numerical results in the linear case.

1.3. Outline. The meshes involved in the construction of the scheme are described in section 2. Some discrete divergence $\operatorname{div}^{\mathcal{T}}$ and gradient $\nabla^{\mathcal{T}}$ operators are 
defined in section 3, that are proved to verify a discrete duality property similar to the Green formula in section 4. The approximation scheme for the nonlinear elliptic equation (1.1) stated in section 5 reads

$$
-\operatorname{div}^{\mathcal{T}}\left(\varphi^{\mathcal{D}}\left(\nabla^{\mathcal{T}} u^{\mathcal{T}}\right)\right)=f^{\mathcal{T}}
$$

where $\mathcal{T}$ is the set of the three finite volume meshes and $\mathcal{D}$ is the set of the diamond cells. The discrete divergence operator maps functions piecewise constant on the diamond cells in $\mathcal{D}$ to functions piecewise constant on the control volumes in $\mathcal{T}$, while the discrete gradient maps functions piecewise constant on the control volumes to functions piecewise constant on the diamond cells. The main properties of our scheme, well-posedness, a priori estimates and some error estimates, are inherited from the discrete duality property and assumptions (1.2), (1.3) and (1.4) exposed in section 6 . The numerical experiments are presented in section 7 .

2. Construction of the meshes. Consider a usual finite volume mesh $\mathcal{M}$ called the primary mesh. We construct two additional finite volume meshes, with control volumes respectively around the vertices and the faces and edges of the primary mesh. They are denoted by $\mathcal{N}$ and $\mathcal{F E}$. The diamond cells $\mathrm{D}$ are defined in order to contain one part of each of the interfaces between control volumes of the three meshes, so that three finite differences are available inside $\mathrm{D}$ to construct the discrete gradient of $u$.

The mesh $\mathcal{T}$ is the triple $(\mathcal{M}, \mathcal{N}, \mathcal{F} \mathcal{E})$ of meshes on $\Omega$, defined below (see figure 2.2). We refer as $\mathrm{C} \in \mathcal{T}$ for any of the volumes in $\mathcal{M} \cup \mathcal{N} \cup \mathcal{F E}$.

2.1. The primary mesh. The mesh $\mathcal{M}$ is a set of open disjoint polyhedral control volumes $\mathrm{K} \subset \Omega$ such that $\cup \overline{\mathrm{K}}=\bar{\Omega}$. The interfaces $\overline{\mathrm{K}} \cap \overline{\mathrm{L}}$ of these control volumes $^{1}$ are denoted by $\mathrm{F}=\overline{\mathrm{K}} \cap \overline{\mathrm{L}}$ as well as the remaining boundary faces $\mathrm{F}=\partial \mathrm{K} \cap \partial \Omega$. They are all supposed to be polygonal. The vertices of theses faces $\mathrm{F}$ are denoted by $x_{\mathrm{A}}$ and called the vertices of $\mathcal{M}$, while the edges of these faces are called the edges of $\mathcal{M}$ and denoted by $\mathrm{E}$.

We associate to each cell $\mathrm{K}$ a point $x_{\mathrm{K}} \in \mathrm{K}$, to each face $\mathrm{F}$ a point $x_{\mathrm{F}} \in \mathrm{F}$ and finally to each edge $\mathrm{E}$ a point $x_{\mathrm{E}} \in \mathrm{E}$. They are for example the isobarycenters of $\mathrm{K}$, F, E.

For each boundary face $\mathrm{F} \subset \partial \Omega$, we introduce a degenerate boundary control volume $\mathrm{K}$ reduced to the face $F$, with center $x_{\mathrm{K}}=x_{\mathrm{F}}$. The set of these degenerated boundary control volume is denoted by $\partial \mathcal{M}$.

DEFINITION 2.1. We define the relation $\prec$ between respectively the vertices and edges, edges and faces, faces and control volumes as "belongs to the boundary to". In other words

$$
x_{\mathrm{A}} \prec x_{\mathrm{E}} \prec x_{\mathrm{F}} \prec x_{\mathrm{K}} \quad \text { means } \quad x_{\mathrm{A}} \subset \partial \mathrm{E}, \quad \mathrm{E} \subset \partial \mathrm{F}, \quad \mathrm{F} \subset \partial \mathrm{K} .
$$

This relation is useful to describe, for instance, the subset of the edges that are connected to a given node $x_{\mathrm{A}}$ (e.g. $\left\{x_{\mathrm{E}}: x_{\mathrm{A}} \prec x_{\mathrm{E}}\right\}$ ), or the subset of the edges that are boundaries of a face F (e.g. $\left\{x_{\mathrm{E}}: x_{\mathrm{E}} \prec x_{\mathrm{F}}\right\}$ ), etc.

2.2. The diamond cells. Consider an edge $\mathrm{E}$ of a face $\mathrm{F}: x_{\mathrm{E}} \prec x_{\mathrm{F}}$. The edge E has two endpoints denoted by $x_{\mathrm{A}}$ and $x_{\mathrm{B}}$ and two neighbouring control volumes in $\mathcal{M} \cup \partial \mathcal{M}$ denoted by $\mathrm{K}$ and $\mathrm{L}$. Using the relation $\prec$, they are defined by

$$
\left(\begin{array}{c}
x_{\mathrm{A}} \\
x_{\mathrm{B}}
\end{array}\right) \prec x_{\mathrm{E}} \prec x_{\mathrm{F}} \prec\left(\begin{array}{c}
x_{\mathrm{K}} \\
x_{\mathrm{L}}
\end{array}\right) .
$$

\footnotetext{
${ }^{1}$ when they have a non zero $d-1$ dimensional measure
} 
Each of the pairs (E, F) with $x_{\mathrm{E}} \prec x_{\mathrm{F}}$ defines a diamond cell that is the polyhedra with vertices $x_{\mathrm{A}}, x_{\mathrm{B}}, x_{\mathrm{E}}, x_{\mathrm{F}}, x_{\mathrm{K}}, x_{\mathrm{L}}$. More specifically, it is denoted by $\mathrm{D}(\mathrm{E}, \mathrm{F})$ or simply $\mathrm{D}$ and given by

$$
\mathrm{D}=\mathrm{D}(\mathrm{E}, \mathrm{F})=\operatorname{hull}\left(x_{\mathrm{A}}, x_{\mathrm{F}}, x_{\mathrm{B}}, x_{\mathrm{K}}\right) \cup \operatorname{hull}\left(x_{\mathrm{A}}, x_{\mathrm{F}}, x_{\mathrm{B}}, x_{\mathrm{L}}\right)
$$

where hull( $(\cdot)$ denotes the convex hull of a set of points. The set of diamond cells is called the diamond mesh and denoted by $\mathcal{D}$. We associate to each diamond cell a point $x_{\mathrm{D}} \in \mathrm{D}$ called the "center" of D and defined as the midpoint between $x_{\mathrm{E}}$ and $x_{\mathrm{F}}$ :

$$
x_{\mathrm{D}}=\frac{1}{2}\left(x_{\mathrm{E}}+x_{\mathrm{F}}\right) .
$$

With the center $x_{\mathrm{D}}$ of $\mathrm{D}(\mathrm{E}, \mathrm{F})$, the diamond cell can be seen as a collection of eight tetrahedra sharing $x_{\mathrm{D}}$ as a common vertex, with three more vertices chosen within the pairs $\left(x_{\mathrm{A}}, x_{\mathrm{B}}\right),\left(x_{\mathrm{E}}, x_{\mathrm{F}}\right)$ and $\left(x_{\mathrm{K}}, x_{\mathrm{L}}\right)$ which we denote by

$$
\mathrm{D}=\operatorname{hull}\left(x_{\mathrm{D}},\left(\begin{array}{l}
x_{\mathrm{A}} \\
x_{\mathrm{B}}
\end{array}\right),\left(\begin{array}{c}
x_{\mathrm{E}} \\
x_{\mathrm{F}}
\end{array}\right),\left(\begin{array}{c}
x_{\mathrm{K}} \\
x_{\mathrm{L}}
\end{array}\right)\right) .
$$

Remark: If $x_{\mathrm{F}} \in \partial \Omega$ then the diamond cell D degenerates into only four tetrahedra, because $x_{\mathrm{F}}=x_{\mathrm{L}}$, namely

$$
\mathrm{D}=\operatorname{hull}\left(x_{\mathrm{D}},\left(\begin{array}{c}
x_{\mathrm{A}} \\
x_{\mathrm{B}}
\end{array}\right),\left(\begin{array}{c}
x_{\mathrm{E}} \\
x_{\mathrm{F}}
\end{array}\right), x_{\mathrm{K}}\right) .
$$

The four remaining tetrahedra degenerate into the four triangles

$$
\operatorname{hull}\left(x_{\mathrm{D}},\left(\begin{array}{l}
x_{\mathrm{A}} \\
x_{\mathrm{B}}
\end{array}\right),\left(\begin{array}{l}
x_{\mathrm{E}} \\
x_{\mathrm{F}}
\end{array}\right)\right)
$$

that are subsets of $\partial \Omega$.

The six vertices $x_{\mathrm{K}}, x_{\mathrm{L}}, x_{\mathrm{A}}, x_{\mathrm{B}}$ and $x_{\mathrm{E}}, x_{\mathrm{F}}$ of the diamond cell $\mathrm{D}(\mathrm{E}, \mathrm{F})$ are supposed to be ordered in such a way that

$$
\Delta_{\mathrm{EF}}:=\operatorname{det}\left(x_{\mathrm{B}}-x_{\mathrm{A}}, x_{\mathrm{F}}-x_{\mathrm{E}}, x_{\mathrm{L}}-x_{\mathrm{K}}\right)>0 .
$$

With this orientation the measure of $\mathrm{D}$ is

$$
|\mathrm{D}|=\frac{1}{6} \Delta_{\mathrm{EF}} .
$$

2.3. The node mesh. A control volume denoted by A is associated to each vertex $x_{\mathrm{A}}$ of the mesh. It can be defined as follows by gathering the four tetrahedra that share $x_{\mathrm{A}}$ as a common vertex within each diamond cell $\mathrm{D}$ for which $x_{\mathrm{A}}$ is a vertex: consider first the set

$$
\mathrm{D}_{\mathrm{A}}=\left\{\mathrm{D}(\mathrm{E}, \mathrm{F}) \in \mathcal{D}, \text { with } x_{\mathrm{A}} \prec x_{\mathrm{E}} \prec x_{\mathrm{F}}\right\} ;
$$

The node cell $\mathrm{A}$ is

$$
\mathrm{A}=\underset{\mathrm{D}(\mathrm{E}, \mathrm{F}) \in \mathrm{D}_{\mathrm{A}}}{\cup} \operatorname{hull}\left(x_{\mathrm{D}}, x_{\mathrm{A}},\left(\begin{array}{c}
x_{\mathrm{E}} \\
x_{\mathrm{F}}
\end{array}\right),\left(\begin{array}{c}
x_{\mathrm{K}} \\
x_{\mathrm{L}}
\end{array}\right)\right) .
$$

This definition is valid for both the interior and boundary vertices $x_{\mathrm{A}}$. The set of the node cells associated to the interior vertices $x_{\mathrm{A}} \in \Omega$ is denoted by $\mathcal{N}$ and the set of the node cells associated to the boundary vertices $x_{\mathrm{A}} \in \partial \Omega$ is denoted by $\partial \mathcal{N}$ :

$$
\mathcal{N}=\left\{\mathrm{A}, x_{\mathrm{A}} \in \Omega\right\}, \quad \partial \mathcal{N}=\left\{\mathrm{A}, x_{\mathrm{A}} \in \partial \Omega\right\} .
$$



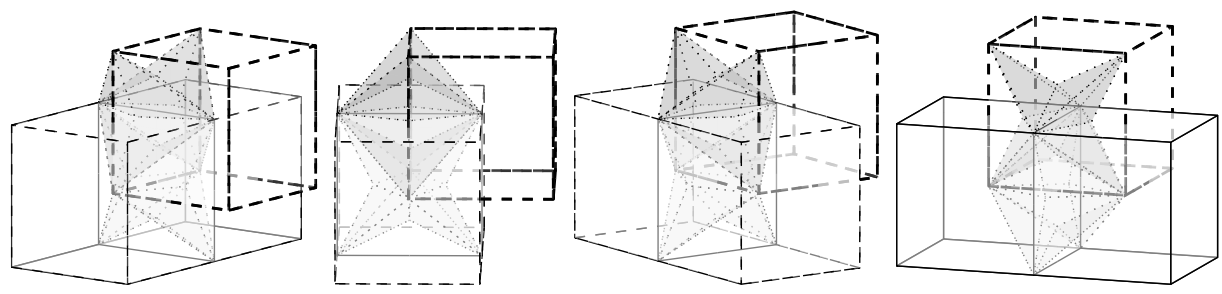

(a) The 3 meshes.

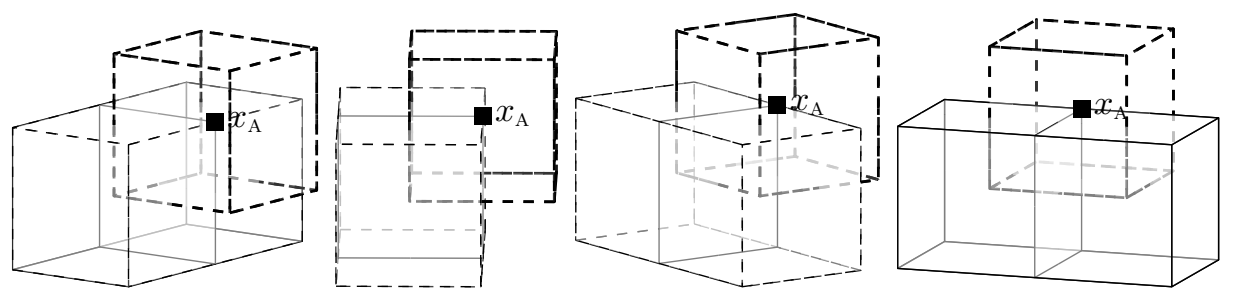

(b) Primary mesh $\mathcal{M}$ and a node cell associated to $x_{\mathrm{A}}$.
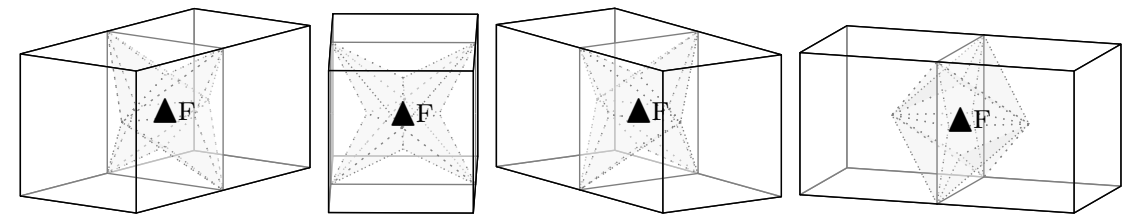

(c) Primary mesh $\mathcal{M}$ and a face cell associated to $x_{\mathrm{F}}$.
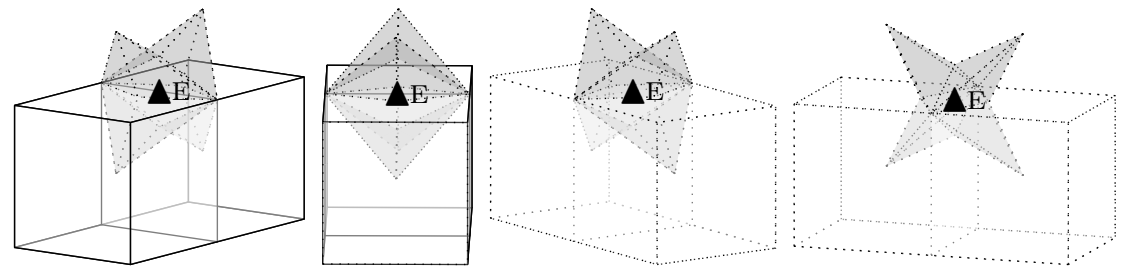

(d) Primary mesh $\mathcal{M}$ and an edge cell associated to $x_{\mathrm{E}}$.

Figure 2.1. 3D views of the 3 meshes for a Cartesian grid.

2.4. The face+edge mesh. A control volume is associated to each center $x_{\mathrm{F}}$ of the faces $\mathrm{F}$ and to each center $x_{\mathrm{E}}$ of the edges E. For sake of simplicity and because we shall no more need to refer to the face or the edge directly, we can securely use the same notations, $\mathrm{E}$ and $\mathrm{F}$, to denote the control volumes associated to $x_{\mathrm{E}}$ and $x_{\mathrm{F}}$ : from now on, $\mathrm{E}$ and $\mathrm{F}$ denote control volumes.

They are defined like the node cells A: consider first the sets

$$
\mathrm{D}_{\mathrm{E}}=\left\{\mathrm{D}(\mathrm{E}, \mathrm{F}) \in \mathcal{D} \text {, with } x_{\mathrm{E}} \prec x_{\mathrm{F}}\right\}, \quad \mathrm{D}_{\mathrm{F}}=\left\{\mathrm{D}(\mathrm{E}, \mathrm{F}) \in \mathcal{D} \text {, with } x_{\mathrm{E}} \prec x_{\mathrm{F}}\right\}
$$

of the diamond cells that share $x_{\mathrm{E}}$ or $x_{\mathrm{F}}$ as a vertex; the face and edge cells $\mathrm{E}$ and $\mathrm{F}$ are defined by

$$
\mathrm{E} \underset{\mathrm{D}(\mathrm{E}, \mathrm{F}) \in \mathrm{D}_{\mathrm{E}}}{\cup} \operatorname{hull}\left(x_{\mathrm{D}},\left(\begin{array}{c}
x_{\mathrm{A}} \\
x_{\mathrm{B}}
\end{array}\right), x_{\mathrm{E}},\left(\begin{array}{c}
x_{\mathrm{K}} \\
x_{\mathrm{L}}
\end{array}\right)\right), \mathrm{F} \underset{\mathrm{D}(\mathrm{E}, \mathrm{F}) \in \mathrm{D}_{\mathrm{F}}}{\cup} \operatorname{hull}\left(x_{\mathrm{D}},\left(\begin{array}{c}
x_{\mathrm{A}} \\
x_{\mathrm{B}}
\end{array}\right), x_{\mathrm{F}},\left(\begin{array}{c}
x_{\mathrm{K}} \\
x_{\mathrm{L}}
\end{array}\right)\right) .
$$

This definition is valid for both the interior and boundary edges and faces. It is 
defined $\mathcal{F E}$ and $\partial \mathcal{F E}$ as

$$
\mathcal{F} \mathcal{E}=\left\{\mathrm{E}, x_{\mathrm{E}} \in \Omega\right\} \cup\left\{\mathrm{F}, x_{\mathrm{F}} \in \Omega\right\}, \quad \partial \mathcal{F} \mathcal{E}=\left\{\mathrm{E}, x_{\mathrm{E}} \in \partial \Omega\right\} \cup\left\{\mathrm{F}, x_{\mathrm{F}} \in \partial \Omega\right\} .
$$

For sake of clarity in the exposition, the set $\mathcal{F} \mathcal{E}$ is split into $\mathcal{E}$ and $\mathcal{F}$, respectively the edge cells, associated to the $x_{\mathrm{E}}$, and the face cells, associated to the $x_{\mathrm{F}}$.

$\mathrm{k}$ Using the same notation, it can be defined the sets

$$
\mathrm{D}_{\mathrm{K}}=\left\{\mathrm{D}(\mathrm{E}, \mathrm{F}) \in \mathcal{D}, \text { with } x_{\mathrm{E}} \prec x_{\mathrm{K}}\right\}
$$

and then the control volumes $\mathrm{K}$ of the primary mesh $\mathcal{M}$ are simply

$$
\mathrm{K}=\underset{\mathrm{D}(\mathrm{E}, \mathrm{F}) \in \mathrm{D}_{\mathrm{K}}}{\cup} \operatorname{hull}\left(x_{\mathrm{D}},\left(\begin{array}{c}
x_{\mathrm{A}} \\
x_{\mathrm{B}}
\end{array}\right),\left(\begin{array}{c}
x_{\mathrm{E}} \\
x_{\mathrm{F}}
\end{array}\right), x_{\mathrm{K}}\right) .
$$

\subsection{Interfaces within the three meshes.}

The DDFV scheme is based on three finite volume formulations on the meshes $\mathcal{M}, \mathcal{N}$ and $\mathcal{F} \mathcal{E}$. The interfaces between pairs of control volumes of these three meshes are described in terms of triangles inside each diamond cell D.

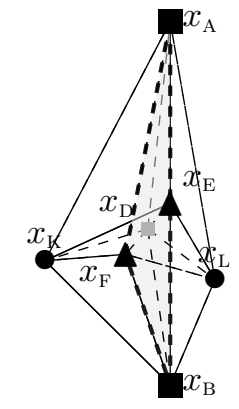

(a) Interface $S_{\mathrm{KL}}$.

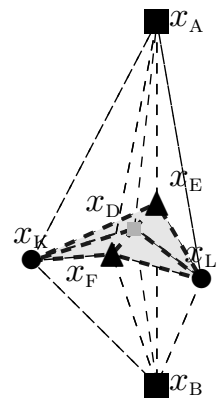

(b) Interface $S_{\mathrm{AB}}$.

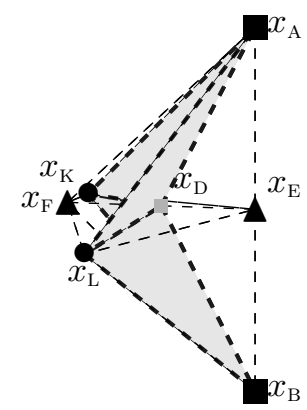

(c) Interface $S_{\mathrm{EF}}$.

Figure 2.2. Interfaces in a diamond cell.

Lemma 2.2. For any diamond cell $\mathrm{D}$ with vertices $x_{\mathrm{K}}, x_{\mathrm{L}}, x_{\mathrm{A}}, x_{\mathrm{B}}, x_{\mathrm{E}}, x_{\mathrm{F}}$, we can define (see fig: 1.2 or fig: 2.2)

$$
\begin{aligned}
S_{\mathrm{KL}}(\mathrm{D}) & :=\overline{\mathrm{K}} \cap \overline{\mathrm{L}} \cap \mathrm{D}=\operatorname{hull}\left(x_{\mathrm{D}},\left(\begin{array}{c}
x_{\mathrm{A}} \\
x_{\mathrm{B}}
\end{array}\right),\left(\begin{array}{l}
x_{\mathrm{E}} \\
x_{\mathrm{F}}
\end{array}\right)\right), \\
N_{\mathrm{KL}} & =\int_{S_{\mathrm{KL}}} n_{\mathrm{KL}} d s=\frac{1}{2}\left(x_{\mathrm{B}}-x_{\mathrm{A}}\right) \times\left(x_{\mathrm{F}}-x_{\mathrm{E}}\right), \\
S_{\mathrm{AB}}(\mathrm{D}) & :=\overline{\mathrm{A}} \cap \overline{\mathrm{B}} \cap \mathrm{D}=\operatorname{hull}\left(x_{\mathrm{D}},\left(\begin{array}{c}
x_{\mathrm{E}} \\
x_{\mathrm{F}}
\end{array}\right),\left(\begin{array}{l}
x_{\mathrm{K}} \\
x_{\mathrm{L}}
\end{array}\right)\right), \\
N_{\mathrm{AB}} & =\int_{S_{\mathrm{AB}}} n_{\mathrm{AB}} d s=\frac{1}{2}\left(x_{\mathrm{F}}-x_{\mathrm{E}}\right) \times\left(x_{\mathrm{L}}-x_{\mathrm{K}}\right), \\
S_{\mathrm{EF}}(\mathrm{D}) & :=\overline{\mathrm{E}} \cap \overline{\mathrm{F}} \cap \mathrm{D}=\operatorname{hull}\left(x_{\mathrm{D}},\left(\begin{array}{c}
x_{\mathrm{K}} \\
x_{\mathrm{L}}
\end{array}\right),\left(\begin{array}{l}
x_{\mathrm{A}} \\
x_{\mathrm{B}}
\end{array}\right)\right), \\
N_{\mathrm{EF}} & =\int_{S_{\mathrm{EF}}} n_{\mathrm{E}, \mathrm{F}} d s=\frac{1}{2}\left(x_{\mathrm{L}}-x_{\mathrm{K}}\right) \times\left(x_{\mathrm{B}}-x_{\mathrm{A}}\right),
\end{aligned}
$$


where $n_{\mathrm{KL}}$ stands for the unit normal to $S_{\mathrm{KL}}(\mathrm{D})$ oriented from $\mathrm{K}$ to $\mathrm{L}, n_{\mathrm{AB}}$ stands for the unit normal to $S_{\mathrm{AB}}(\mathrm{D})$ oriented from $\mathrm{A}$ to $\mathrm{B}$ and $n_{\mathrm{E}, \mathrm{F}}$ stands for the unit normal to $S_{\mathrm{EF}}(\mathrm{D})$ oriented from $\mathrm{E}$ to $\mathrm{F}$.

Furthermore, note that

$$
N_{\mathrm{KL}} \cdot\left(x_{\mathrm{L}}-x_{\mathrm{K}}\right)=N_{\mathrm{AB}} \cdot\left(x_{\mathrm{B}}-x_{\mathrm{A}}\right)=N_{\mathrm{EF}} \cdot\left(x_{\mathrm{F}}-x_{\mathrm{E}}\right)=\frac{1}{2} \Delta_{\mathrm{EF}}=3|\mathrm{D}| .
$$

Proof. A straightforward computation shows that the interfaces between pairs of control volumes are indeed made of triangles, and their average normal can be expressed using simple vector products, so that eq. (2.11) is obvious. $\square$

Finally, the boundaries of the interior control volumes $\mathrm{K} \in \mathcal{M}, \mathrm{A} \in \mathcal{N}, \mathrm{E}, \mathrm{F} \in \mathcal{F E}$ can be expressed in terms of the $S_{\mathrm{KL}}(\mathrm{D}), S_{\mathrm{EF}}(\mathrm{D}), S_{\mathrm{AB}}(\mathrm{D})$ :

$$
\begin{aligned}
\partial \mathrm{K}=\bigcup_{\mathrm{D} \in \mathrm{D}_{\mathrm{K}}} S_{\mathrm{KL}}(\mathrm{D}), & \partial \mathrm{A}=\underset{\mathrm{D} \in \mathrm{D}_{\mathrm{A}}}{\cup} S_{\mathrm{AB}}(\mathrm{D}), \\
\partial \mathrm{E}=\underset{\mathrm{D} \in \mathrm{D}_{\mathrm{E}}}{\cup} S_{\mathrm{EF}}(\mathrm{D}), & \partial \mathrm{F}=\underset{\mathrm{D} \in \mathrm{D}_{\mathrm{F}}}{\cup} S_{\mathrm{EF}}(\mathrm{D}) .
\end{aligned}
$$

Remark: In the case $\mathrm{A} \in \partial \mathcal{N}$ or $\mathrm{E}$ or $\mathrm{F} \in \partial \mathcal{F} \mathcal{E}$, it is important to note that the boundary $\partial \mathrm{A}, \partial \mathrm{E}$ or $\partial \mathrm{F}$ is not easily described in such a way. Anyway they are not needed since only Dirichlet boundary conditions are used: the scheme writes only on interior control volumes.

2.6. Regularity of the meshes. Given a diamond cell $\mathrm{D} \in \mathcal{D}$, let

$$
\nu_{\mathrm{KL}}=\frac{N_{\mathrm{KL}}}{\left|N_{\mathrm{KL}}\right|}, \quad \nu_{\mathrm{AB}}=\frac{N_{\mathrm{AB}}}{\left|N_{\mathrm{AB}}\right|}, \quad \nu_{\mathrm{EF}}=\frac{N_{\mathrm{EF}}}{\left|N_{\mathrm{EF}}\right|}
$$

denote the unit normals (see eqs. (2.6)-(2.10)) and

$$
G_{\mathrm{D}}=\left(\begin{array}{lll}
\nu_{\mathrm{KL}} \cdot \nu_{\mathrm{KL}} & \nu_{\mathrm{KL}} \cdot \nu_{\mathrm{AB}} & \nu_{\mathrm{KL}} \cdot \nu_{\mathrm{EF}} \\
\nu_{\mathrm{AB}} \cdot \nu_{\mathrm{KL}} & \nu_{\mathrm{AB}} \cdot \nu_{\mathrm{AB}} & \nu_{\mathrm{AB}} \cdot \nu_{\mathrm{EF}} \\
\nu_{\mathrm{EF}} \cdot \nu_{\mathrm{KL}} & \nu_{\mathrm{EF}} \cdot \nu_{\mathrm{AB}} & \nu_{\mathrm{EF}} \cdot \nu_{\mathrm{EF}}
\end{array}\right)
$$

denote the Gram matrix associated to $\left(\nu_{\mathrm{KL}}, \nu_{\mathrm{AB}}, \nu_{\mathrm{EF}}\right)$.

DeFINITION 2.3. The size $h$ of the mesh $\mathcal{T}$ is the maximum diameter of the diamond cells,

$$
h_{\mathrm{D}}=\sup _{x, y \in \mathrm{D}}|x-y|, \quad h=\max _{\mathrm{D} \in \mathcal{D}} h_{\mathrm{D}}
$$

It is assumed that each diamond cell $\mathrm{D}$ contains an open ball $B_{\mathrm{D}}$ of diameter $R_{\mathrm{D}}$ such that $\mathrm{D}$ is star-shaped with respect to $B_{\mathrm{D}}$, that is $\{t x+(1-t) y, t \in(0,1)\} \subset \mathrm{D}$ for any $x \in B_{\mathrm{D}}$ and $y \in \mathrm{D}$.

The constant of regularity of the mesh is any number $c_{r}(\mathcal{T})>0$ such that

$$
\forall \mathrm{D} \in \mathcal{D}, \quad \frac{1}{\lambda_{m}(\mathrm{D})} \leq c_{r}(\mathcal{T}), \quad \frac{|\mathrm{D}|}{\left|\mathrm{D}_{i}\right|} \leq c_{r}(\mathcal{T}), \quad \frac{h_{\mathrm{D}}}{R_{\mathrm{D}}} \leq c_{r}(\mathcal{T})
$$

where $\lambda_{m}(\mathrm{D})$ is the smallest eigenvalue of $G_{\mathrm{D}},\left(\mathrm{D}_{i}\right)_{i=1 \ldots 8}$ are the 8 tetrahedra that compose D (see eq. (2.1)).

\section{The discrete spaces and operators.}


3.1. The discrete spaces. Consider the data $\left(u^{\mathcal{M}}, u^{\mathcal{N}}, u^{\mathcal{F E}}\right)$ of three functions piecewise constant respectively on the $\mathrm{K} \in \mathcal{M}, \mathrm{A} \in \mathcal{N}$ and $\mathrm{E} / \mathrm{F} \in \mathcal{F} \mathcal{E}$ :

$$
u^{\mathcal{M}}=\sum_{\mathrm{K} \in \mathcal{M}} u_{\mathrm{K}} \chi_{\mathrm{K}}, \quad u^{\mathcal{N}}=\sum_{A \in \mathcal{N}} u_{\mathrm{A}} \chi_{A}, \quad u^{\mathcal{F E}}=\sum_{\mathrm{F} \in \mathcal{F}} u_{\mathrm{F}} \chi_{\mathrm{F}}+\sum_{\mathrm{E} \in \mathcal{E}} u \chi_{\mathrm{E}}
$$

The sets of functions piecewise constant on the $K \in \mathcal{M}, \mathrm{A} \in \mathcal{N}$ and $\mathrm{E} / \mathrm{F} \in \mathcal{F E}$ are respectively denoted by $X^{\mathcal{M}}, X^{\mathcal{N}}$ and $X^{\mathcal{F E}}$. They are finite dimensional subspaces of any $L^{q}(\Omega)(q \geq 1)$. The finite volume unknown is an element of the space $X=$ $X^{\mathcal{M}} \times X^{\mathcal{N}} \times X^{\mathcal{F} \bar{E}}$. It is denoted by

$$
u^{\mathcal{T}}=\left(u^{\mathcal{M}}, u^{\mathcal{N}}, u^{\mathcal{F E}}\right)=\left(\left(u_{\mathrm{K}}\right)_{\mathrm{K} \in \mathcal{M}},\left(u_{\mathrm{A}}\right)_{\mathrm{A} \in \mathcal{N}},\left(u_{\mathrm{E}}\right)_{\mathrm{E} \in \mathcal{E}},\left(u_{\mathrm{F}}\right)_{\mathrm{F} \in \mathcal{F}}\right) .
$$

It is supplemented with boundary values

$$
\delta u^{\mathcal{T}}=\left(\left(u_{\mathrm{K}}\right)_{x_{\mathrm{K}} \in \partial \mathcal{M}},\left(u_{\mathrm{A}}\right)_{x_{\mathrm{A}} \in \partial \Omega},\left(u_{\mathrm{E}}\right)_{x_{\mathrm{E}} \in \partial \Omega},\left(u_{\mathrm{F}}\right)_{x_{\mathrm{F}} \in \partial \Omega}\right)
$$

that define a linear space $\partial X$. These boundary values $\delta u^{\mathcal{T}}$ are actually determined by a suitable projection of the boundary data $g$ (see eq. (5.3) below).

The space $X$ is supplied with the natural inner product

$$
\begin{aligned}
\left(u^{\mathcal{T}}, v^{\mathcal{T}}\right)_{X}= & \frac{1}{3}\left(\int_{\Omega} u^{\mathcal{M}} v^{\mathcal{M}}+\int_{\Omega} u^{\mathcal{N}} v^{\mathcal{N}}+\int_{\Omega} u^{\mathcal{F E}} v^{\mathcal{F E}}\right) \\
& =\frac{1}{3}\left(\sum_{\mathrm{K} \in \mathcal{M}} u_{\mathrm{K}} v_{\mathrm{K}}|\mathrm{K}|+\sum_{\mathrm{A} \in \mathcal{N}} u_{\mathrm{A}} v_{\mathrm{A}}|\mathrm{A}|+\sum_{\mathrm{E} \in \mathcal{E}} u_{\mathrm{E}} v_{\mathrm{E}}|\mathrm{E}|+\sum_{\mathrm{F} \in \mathcal{F}} u_{\mathrm{F}} v_{\mathrm{F}}|\mathrm{F}|\right)
\end{aligned}
$$

For this norm, the inequality of Hölder reads:

$$
\begin{aligned}
\left(u^{\mathcal{T}}, v^{\mathcal{T}}\right)_{X} \leq \frac{1}{3}\left(\left\|u^{\mathcal{M}}\right\|_{L^{p^{\prime}}}\left\|v^{\mathcal{M}}\right\|_{L^{p}}+\left\|u^{\mathcal{N}}\right\|_{L^{p^{\prime}}}\left\|v^{\mathcal{N}}\right\|_{L^{p}}+\left\|u^{\mathcal{F} \mathcal{E}}\right\|_{L^{p^{\prime}}}\left\|v^{\mathcal{F} \mathcal{E}}\right\|_{L^{p}}\right) \\
\leq\left\|u^{\mathcal{T}}\right\|_{L^{p^{\prime}}}\left\|v^{\mathcal{T}}\right\|_{L^{p}}
\end{aligned}
$$

with the notation

$$
\left\|u^{\mathcal{T}}\right\|_{L^{p}}=\left(\frac{1}{3}\left(\left\|u^{\mathcal{M}}\right\|_{L^{p}}^{p}+\left\|u^{\mathcal{N}}\right\|_{L^{p}}^{p}+\left\|u^{\mathcal{F} \mathcal{E}}\right\|_{L^{p}}^{p}\right)\right)^{1 / p} .
$$

A linear space of vector fields will be associated to the finite volumes gradient. It is the space $\mathbf{Q}$ of functions piecewise constant on the $\mathrm{D} \in \mathcal{D}$ with value in $\mathbb{R}^{3}$ :

$$
\xi^{\mathcal{D}} \in \mathbf{Q} \Leftrightarrow \xi^{\mathcal{D}}=\sum_{\mathrm{D} \in \mathcal{D}} \xi_{\mathrm{D}} \chi_{\mathrm{D}}, \quad \forall \mathrm{D} \in \mathcal{D}, \xi_{\mathrm{D}} \in \mathbb{R}^{3}
$$

These functions are $\left(L^{q}(\Omega)\right)^{3}$ for any $q \geq 1$ and like for elements $u^{\mathcal{T}} \in X$, an element $\xi^{\mathcal{D}} \in \mathbf{Q}$ may also be denoted by the sequence of its degrees of freedom: $\xi^{\mathcal{D}}=\left(\xi_{\mathrm{D}}\right)_{\mathrm{D} \in \mathcal{D}}$. The space $\mathbf{Q}$ is endowed with the natural inner product

$$
\left(\xi^{\mathcal{D}}, \eta^{\mathcal{D}}\right)_{\mathbf{Q}}=\int_{\Omega} \xi^{\mathcal{D}} \cdot \eta^{\mathcal{D}}=\sum_{\mathrm{D} \in \mathcal{D}} \xi_{\mathrm{D}} \cdot \eta_{\mathrm{D}}|\mathrm{D}|
$$


3.2. The discrete gradient. The discrete gradient is defined for $\left(u^{\mathcal{T}}, \delta u^{\mathcal{T}}\right) \in$ $X \times \partial X$. It is a vector field in $\mathbf{Q}$, denoted by $\nabla_{\delta u}^{\mathcal{T}} u^{\mathcal{T}}=\left(\nabla_{\delta u}^{\mathrm{D}} u^{\mathcal{T}}\right)_{\mathrm{D} \in \mathcal{D}}$ where

$$
\forall \mathrm{D} \in \mathcal{D}, \quad \nabla_{\delta u}^{\mathrm{D}} u^{\mathcal{T}}=\frac{1}{3|\mathrm{D}|}\left(\left(u_{\mathrm{L}}-u_{\mathrm{K}}\right) N_{\mathrm{KL}}+\left(u_{\mathrm{B}}-u_{\mathrm{A}}\right) N_{\mathrm{AB}}+\left(u_{\mathrm{F}}-u_{\mathrm{E}}\right) N_{\mathrm{EF}}\right) .
$$

The mapping $\left(u^{\mathcal{T}}, \delta u^{\mathcal{T}}\right) \in X \times \delta X \mapsto \nabla_{\delta u}^{\mathcal{T}} u^{\mathcal{T}} \in \mathbf{Q}$ is linear with respect to $\left(u^{\mathcal{T}}, \delta u^{\mathcal{T}}\right)$ and we shall need a "homogeneous" gradient, denoted by $\nabla_{0}^{\mathcal{T}}: u^{\mathcal{T}} \in X \mapsto \nabla_{0}^{\mathcal{T}} u^{\mathcal{T}} \in \mathbf{Q}$ defined by eq. (3.5) with $\delta u^{\mathcal{T}}=0_{\delta X}$. It is a linear mapping from $X$ onto $\mathbf{Q}$.

LEMma 3.1 (Properties of the discrete gradient). By construction, for each diamond cell $\mathrm{D} \in \mathcal{D}$,

1. the vector $\nabla_{\delta u}^{\mathrm{D}} u^{\mathcal{T}}$ is the unique vector of $\mathbb{R}^{3}$ such that

$\nabla_{\delta u}^{\mathrm{D}} u^{\mathcal{T}} \cdot\left(x_{\mathrm{L}}-x_{\mathrm{K}}\right)=u_{\mathrm{L}}-u_{\mathrm{K}}, \quad \nabla_{\delta u}^{\mathrm{D}} u^{\mathcal{T}} \cdot\left(x_{\mathrm{B}}-x_{\mathrm{A}}\right)=u_{\mathrm{B}}-u_{\mathrm{A}}, \quad \nabla_{\delta u}^{\mathrm{D}} u^{\mathcal{T}} \cdot\left(x_{\mathrm{F}}-x_{\mathrm{E}}\right)=u_{\mathrm{F}}-u_{\mathrm{E}}$,

2. if $u(x)=u_{0}+p \cdot x$ is an affine function and $u_{\mathrm{K}}=u\left(x_{\mathrm{K}}\right), u_{\mathrm{L}}=u\left(x_{\mathrm{L}}\right)$, $u_{\mathrm{E}}=u\left(x_{\mathrm{E}}\right), u_{\mathrm{F}}=u\left(x_{\mathrm{F}}\right), u_{\mathrm{A}}=u\left(x_{\mathrm{A}}\right), u_{\mathrm{B}}=u\left(x_{\mathrm{B}}\right)$, then

$$
\nabla_{\delta u}^{\mathrm{D}} u^{\mathcal{T}}=\nabla u=p \quad \text { in } \mathbb{R}^{3} .
$$

Proof. The first point is a consequence of the relation (2.11) and the second point is a consequence of the first one.

3.3. The discrete divergence. The discrete divergence is defined for a vector field $\xi^{\mathcal{D}} \in \mathbf{Q}$. It is an element of $X$ denoted by

$$
\operatorname{div}^{\mathcal{T}} \xi^{\mathcal{D}}=\left(\operatorname{div}^{\mathcal{M}} \xi^{\mathcal{D}}, \operatorname{div}^{\mathcal{N}} \xi^{\mathcal{D}}, \operatorname{div}^{\mathcal{F} \mathcal{E}} \xi^{\mathcal{D}}\right) \in X
$$

where

$$
\begin{gathered}
\operatorname{div}^{\mathcal{M}} \xi^{\mathcal{D}}=\left(\operatorname{div}_{\mathrm{K}} \xi^{\mathcal{D}}\right)_{\mathrm{K} \in \mathcal{M}}, \quad \operatorname{div}^{\mathcal{N}} \xi^{\mathcal{D}}=\left(\operatorname{div}_{\mathrm{A}} \xi^{\mathcal{D}}\right)_{\mathrm{A} \in \mathcal{N}} \\
\operatorname{div}^{\mathcal{F E}} \xi^{\mathcal{D}}=\left\{\left(\operatorname{div}_{\mathrm{E}} \xi^{\mathcal{D}}\right)_{\mathrm{E} \in \mathcal{E}},\left(\operatorname{div}_{\mathrm{F}} \xi^{\mathcal{D}}\right)_{\mathrm{F} \in \mathcal{F}}\right\}
\end{gathered}
$$

For any control volume denoted in general by $\mathrm{C}=\mathrm{K}, \mathrm{A}, \mathrm{E}, \mathrm{F} \in \mathcal{T}$, the discrete divergence is the finite volume one:

$$
|\mathrm{C}| \operatorname{div}_{\mathrm{C}} \xi^{\mathcal{D}}=\int_{\partial \mathrm{C}} \xi^{\mathcal{D}}(x) \cdot n_{\mathrm{C}}(x) d \sigma(x),
$$

where $n_{\mathrm{C}}$ is the unit normal to $\partial \mathrm{C}$ outward of C. Because of lemma 2.2 and equalities (2.12) and (2.13), these discrete divergences read

$$
\begin{gathered}
|\mathrm{K}| \operatorname{div}_{\mathrm{K}} \xi^{\mathcal{D}}=\sum_{\mathrm{D} \in \mathrm{D}_{\mathrm{K}}} \xi_{\mathrm{D}} \cdot N_{\mathrm{KL}}, \quad|\mathrm{A}| \operatorname{div}_{\mathrm{A}} \xi^{\mathcal{D}}=\sum_{\mathrm{D} \in \mathrm{D}_{\mathcal{A}}} \xi_{\mathrm{D}} \cdot N_{\mathrm{AB}}, \\
|\mathrm{E}| \operatorname{div}_{\mathrm{E}} \xi^{\mathcal{D}}=\sum_{\mathrm{D} \in \mathrm{D}_{\mathrm{E}}} \xi_{\mathrm{D}} \cdot N_{\mathrm{EF}}, \quad|\mathrm{F}| \operatorname{div}_{\mathrm{F}} \xi^{\mathcal{D}}=\sum_{\mathrm{D} \in \mathrm{D}_{\mathrm{F}}} \xi_{\mathrm{D}} \cdot\left(-N_{\mathrm{EF}}\right) .
\end{gathered}
$$

\section{Properties of the Discrete operators.}

4.1. The discrete duality relationship. We first state a discrete version of the Green formula in the spaces $W^{1, p^{\prime}}(\Omega)$ and $W_{0}^{1, p}(\Omega)$ :

$$
\langle\operatorname{div} q, u\rangle_{L^{p^{\prime}}, L^{p}}+\langle q, \nabla u\rangle_{\left(L^{p^{\prime}}\right)^{3},\left(L^{p}\right)^{3}}=0, \forall u \in W_{0}^{1, p}(\Omega), \forall q \in\left(W^{1, p^{\prime}}(\Omega)\right)^{3} .
$$


Theorem 4.1 (Discrete duality identity). For any $u^{\mathcal{T}} \in X$ and $\xi^{\mathcal{D}} \in \mathbf{Q}$, the homogeneous gradient $\nabla_{0}^{\mathcal{T}} u^{\mathcal{T}}$ and divergence $\operatorname{div}^{\mathcal{T}} \xi^{\mathcal{D}}$ verify the discrete duality relation

$$
\left(\operatorname{div}^{\mathcal{T}} \xi^{\mathcal{D}}, u^{\mathcal{T}}\right)_{X}+\left(\xi^{\mathcal{D}}, \nabla_{0}^{\mathcal{T}} u^{\mathcal{T}}\right)_{\mathbf{Q}}=0
$$

Proof. From the eqs. (3.7) and (3.8) of the divergence and (3.1) of the inner product in $X$, it is computed:

$$
\begin{aligned}
\left(\operatorname{div}^{\mathcal{T}} \xi^{\mathcal{D}}, u^{\mathcal{T}}\right)_{X}= & \frac{1}{3}\left(\sum_{\mathrm{K} \in \mathcal{M}} \sum_{\mathrm{D} \in \mathrm{D}_{\mathrm{K}}} \xi_{\mathrm{D}} \cdot N_{\mathrm{KL}} u_{\mathrm{K}}+\sum_{\mathrm{A} \in \mathcal{N}} \sum_{\mathrm{D} \in \mathrm{D}_{\mathrm{A}}} \xi_{\mathrm{D}} \cdot N_{\mathrm{AB}} u_{\mathrm{A}}\right. \\
& \left.\quad+\sum_{\mathrm{F} \in \mathcal{F}} \sum_{\mathrm{D} \in \mathrm{D}_{\mathrm{F}}} \xi_{\mathrm{D}} \cdot\left(-N_{\mathrm{EF}}\right) u_{\mathrm{F}}+\sum_{\mathrm{E} \in \mathcal{E}} \sum_{\mathrm{D} \in \mathrm{D}_{\mathrm{E}}} \xi_{\mathrm{D}} \cdot N_{\mathrm{EF}} u_{\mathrm{E}}\right) \\
= & -\frac{1}{3} \sum_{\mathrm{D} \in \mathcal{D}} \xi_{\mathrm{D}} \cdot\left(N_{\mathrm{KL}}\left(u_{\mathrm{L}}-u_{\mathrm{K}}\right)+N_{\mathrm{AB}}\left(u_{\mathrm{B}}-u_{\mathrm{A}}\right)+N_{\mathrm{EF}}\left(u_{\mathrm{F}}-u_{\mathrm{E}}\right)\right) \\
= & -\sum_{\mathrm{D} \in \mathcal{D}}|\mathrm{D}| \xi_{\mathrm{D}} \cdot \nabla^{\mathrm{D}} u^{\mathcal{T}}=-\left(\xi^{\mathcal{D}}, \nabla^{\mathcal{T}} u^{\mathcal{T}}\right)_{\mathbf{Q}},
\end{aligned}
$$

using also eqs. (3.5) and (3.4) for the discrete gradient and the inner product in $\mathbf{Q}$, and with the homogeneous Dirichlet condition $\delta u^{\mathcal{T}}=0_{\delta X}$.

4.2. The inequality of Poincaré. The discrete spaces $X \times \delta X$ and $X$ (or $X \times$ $\{0\})$ can be interpreted as discrete counterparts of $W^{1, p}(\Omega)$ and $W_{0}^{1, p}(\Omega)$ respectively. For homogeneous Dirichlet boundary data, that is using $\nabla_{0}^{\mathcal{T}}$ in $X$, it is expected that $\nabla_{0}^{\mathcal{T}} u^{\mathcal{T}}=0 \Rightarrow u^{\mathcal{T}}=0$ and that a discrete inequality of Poincaré holds.

Theorem 4.2 (Inequality of Poincaré). There exists a constant $C>0$ depending on $c_{r}(\mathcal{T})$ such that

$$
\forall u^{\mathcal{T}} \in X, \quad\left\|u^{\mathcal{T}}\right\|_{L^{p}} \leq C\left\|\nabla_{0}^{\mathcal{T}} u^{\mathcal{T}}\right\|_{L^{p}}
$$

Proof. The proof makes use of the notations from eqs. (2.14) and (2.15) for the unit normals inside a diamond D, the associated Gram matrix and its smallest eigenvalue $\lambda_{m}(\mathrm{D})$. For sake of simplicity, consider the notations

$$
a_{\mathrm{KL}}=\left(u_{\mathrm{L}}-u_{\mathrm{K}}\right) \frac{\left|N_{\mathrm{KL}}\right|}{3|\mathrm{D}|}, \quad a_{\mathrm{AB}}=\left(u_{\mathrm{B}}-u_{\mathrm{A}}\right) \frac{\left|N_{\mathrm{AB}}\right|}{3|\mathrm{D}|}, \quad a_{\mathrm{EF}}=\left(u_{\mathrm{F}}-u_{\mathrm{E}}\right) \frac{\left|N_{\mathrm{EF}}\right|}{3|\mathrm{D}|} .
$$

The expression of the discrete gradient now reads $\nabla_{\delta u}^{\mathrm{D}} u^{\mathcal{T}}=a_{\mathrm{KL}} \nu_{\mathrm{KL}}+a_{\mathrm{AB}} \nu_{\mathrm{AB}}+a_{\mathrm{EF}} \nu_{\mathrm{EF}}$ and then

$$
\left|\nabla_{0}^{\mathrm{D}} u^{\mathcal{T}}\right|^{2}=a^{T} G_{\mathrm{D}} a \geq \lambda_{m}(\mathrm{D}) \sum_{i=1}^{3}\left|a_{i}\right|^{2},
$$

where $a=\left(a_{\mathrm{KL}}, a_{\mathrm{AB}}, a_{\mathrm{EF}}\right)$. As a consequence, $\left|a_{i}\right|^{2} \leq 1 / \lambda_{m}(\mathrm{D})\left|\nabla_{0}^{\mathrm{D}} u^{\mathcal{T}}\right|^{2}$, and then

$$
\left|\nabla_{0}^{\mathrm{D}} u^{\mathcal{T}}\right|^{p} \geq \frac{\lambda_{m}(\mathrm{D})^{p / 2}}{3}\left(\left|u_{\mathrm{L}}-u_{\mathrm{K}}\right|^{p} \frac{\left|N_{\mathrm{KL}}\right|^{p}}{3^{p}|\mathrm{D}|^{p}}+\left|u_{\mathrm{B}}-u_{\mathrm{A}}\right|^{p} \frac{\left|N_{\mathrm{AB}}\right|^{p}}{3^{p}|\mathrm{D}|^{p}}+\left|u_{\mathrm{F}}-u_{\mathrm{E}}\right|^{p} \frac{\left|N_{\mathrm{EF}}\right|^{p}}{3^{p}|\mathrm{D}|^{p}}\right) .
$$


It remains to prove that there exists a constant $C>0$ depending on the regularity of the meshes $c_{r}(\mathcal{T})$ and on $\Omega$ such that

$$
\int_{\Omega}\left|u^{\mathcal{M}}(x)\right|^{p} d x \leq C \sum_{\mathrm{D} \in \mathcal{D}}\left|u_{\mathrm{L}}-u_{\mathrm{K}}\right|^{p} \frac{\left|N_{\mathrm{KL}}\right|^{p}}{3^{p}|\mathrm{D}|^{p}}|\mathrm{D}|,
$$

and similarly for the two other finite differences in the above inequality. The usual VF4 computation from [14] yields such a result (see also [4] for the $L^{p}$ case).

\section{The finite volume scheme.}

5.1. Formulation of the scheme. The Discrete Duality Finite Volume scheme is obtained by integrating equation (1.1) on all the control volumes of the three meshes, $\mathrm{K} \in \mathcal{M}, \mathrm{A} \in \mathcal{N}, \mathrm{E} \in \mathcal{E}$ and $\mathrm{F} \in \mathcal{F}[16,10,3]:$ the exact solution $u$ verifies for all $\mathrm{c} \in \mathcal{T}$ (ie $\mathrm{C}=\mathrm{K}, \mathrm{A}, \mathrm{E}, \mathrm{F})$ :

$$
-\int_{\partial \mathrm{C}} \varphi(s, \nabla u(s)) \cdot n_{\mathrm{C}} d s=\int_{\mathrm{C}} f(x) d x .
$$

For any diamond cell $\mathrm{D} \in \mathcal{D}$, consider the spatial approximation $\varphi_{\mathrm{D}}: \mathbb{R}^{3} \rightarrow \mathbb{R}^{3}$ of the flux $\varphi$ defined by

$$
\forall \mathrm{D} \in \mathcal{D}, \forall \xi \in \mathbb{R}^{3}, \quad \varphi_{\mathrm{D}}(\xi)=\frac{1}{|\mathrm{D}|} \int_{\mathrm{D}} \varphi(z, \xi) d z .
$$

The flux $\varphi(\cdot, \nabla u(\cdot))$ is approximated by the vector field in $\mathbf{Q}$ defined by

$$
\varphi^{\mathcal{D}}\left(\nabla_{\delta u}^{\mathcal{T}} u^{\mathcal{T}}\right)=\left(\varphi_{\mathrm{D}}\left(\nabla_{\delta u}^{\mathrm{D}} u^{\mathcal{T}}\right)\right)_{\mathrm{D} \in \mathcal{D}} \in \mathbf{Q},
$$

where $\delta u^{\mathcal{T}}=\mathbf{p}^{\mathcal{T}} g \in \partial X$ is given by a pointwise projection of the data $g$ on the $x_{\mathrm{K}}$, $x_{\mathrm{A}}, x_{\mathrm{E}}$ and $x_{\mathrm{F}}$ on $\partial \Omega$,

$$
\forall g \in C^{0}(\partial \Omega), \quad \mathbf{p}^{\mathcal{T}} g=\left(g\left(x_{\mathrm{K}}\right), g\left(x_{\mathrm{A}}\right), g\left(x_{\mathrm{E}}\right), g\left(x_{\mathrm{F}}\right)\right)_{x_{\mathrm{K}} \in \partial \mathcal{M}, x_{\mathrm{A}} \in \partial \mathcal{N}, x_{\mathrm{E}}, x_{\mathrm{F}} \in \partial \mathcal{F} \mathcal{E}}
$$

and $\nabla_{\delta u}^{\mathcal{T}} u^{\mathcal{T}}$ has been defined in section 3.2. With this approximation of the flux and the discrete divergence described in section 3.3, eq. (5.1) reads

$$
-\operatorname{div}^{\mathcal{T}}\left(\varphi^{\mathcal{D}}\left(\nabla_{\delta u}^{\mathcal{T}} u^{\mathcal{T}}\right)\right)=\pi^{\mathcal{T}} f, \quad \delta u^{\mathcal{T}}=\mathbf{p}^{\mathcal{T}} g
$$

where the projection $\pi^{\mathcal{T}} f=\left\{\left(f_{\mathrm{K}}\right)_{\mathrm{K} \in \mathcal{M}},\left(f_{\mathrm{A}}\right)_{\mathrm{A} \in \mathcal{N}},\left(f_{\mathrm{E}}, f_{\mathrm{F}}\right)_{\mathrm{E} \in \mathcal{E}, \mathrm{F} \in \mathcal{F}}\right\} \in X$ is the piecewise constant projection defined by average values,

$$
\begin{gathered}
\forall \mathrm{K} \in \mathcal{M}, \quad f_{\mathrm{K}}=\frac{1}{|\mathrm{~K}|} \int_{\mathrm{K}} f(x) d x, \quad \forall \mathrm{A} \in \mathcal{N}, \quad f_{\mathrm{A}}=\frac{1}{|\mathrm{~A}|} \int_{\mathrm{A}} f(x) d x, \\
\forall \mathrm{E} \in \mathcal{E}, \quad f_{\mathrm{E}}=\frac{1}{|\mathrm{E}|} \int_{\mathrm{E}} f(x) d x, \quad \forall \mathrm{F} \in \mathcal{F}, \quad f_{\mathrm{F}}=\frac{1}{|\mathrm{~F}|} \int_{\mathrm{F}} f(x) d x .
\end{gathered}
$$

For any fixed values $\delta u^{\mathcal{T}} \in \partial X$, eq. (5.4) is a non linear system of $N_{\mathcal{M}}+N_{\mathcal{N}}+N_{\mathcal{F E}}$ equations with $N_{\mathcal{M}}+N_{\mathcal{N}}+N_{\mathcal{F E}}$ unknowns, where $N_{\mathcal{M}}, N_{\mathcal{N}}$ and $N_{\mathcal{F E}}$ are the number of cells, interior vertices and interior faces plus edges of the primal mesh $\mathcal{T}$.

Remark: in the general case, $g \in W^{1-1 / p, p^{\prime}}(\partial \Omega)$ and $f \in W^{-1, p^{\prime}}(\Omega)$, only the projections $\pi^{\mathcal{T}}$ for the right-hand side $f$ and $\mathbf{p}^{\mathcal{T}}$ for the boundary data $g$ has to be modified. For instance $f_{\mathrm{K}}=\left\langle f, \chi_{\mathrm{K}}\right\rangle$ is a natural choice to define $\pi^{\mathcal{T}}$ and the $g_{\mathrm{K}, \mathrm{A}, \ldots}$ would be some average values of $g$ on the boundary. 
5.2. A word on practical implementation. Note that the implementation of such a scheme does not require the construction of the node mesh $\mathcal{N}$ and the face and edges mesh $\mathcal{F E}$ explicitly. If the primal mesh is given with the format

$$
\mathrm{A} \prec \mathrm{E} \prec \mathrm{F} \prec \mathrm{K}
$$

that is a control volume is defined by its faces, a face by its edges and an edge by its vertices, then a diamond cell is easily constructed by referencing its six vertices $x_{\mathrm{A}}, x_{\mathrm{B}}, x_{\mathrm{K}}, x_{\mathrm{L}}, x_{\mathrm{E}}, x_{\mathrm{F}}$. This is a usual structure for simplicial meshes (i.e. tetrahedra in 3D) of $\Omega$.

The expression of the scheme requires to compute the values $N_{\mathrm{KL}}, N_{\mathrm{AB}}, N_{\mathrm{EF}}$ and the measures of the eight tetrahedral cells that compose the diamond cell (see eq. (2.1)). The system of equations involved in the resolution of the scheme can be easily implemented by iterating through the diamond cell structure.

For instance, in the linear case, $\varphi(z, \nabla u(z))=\mathbf{K}(x) \nabla u(x)$ where $\mathbf{K}(x)$ is a uniformly elliptic symmetric matrix, the flux in the diamond cell $\mathrm{D}$ is $\mathbf{K}_{\mathrm{D}} \nabla_{\delta u}^{\mathrm{D}} u^{\mathcal{T}}$ and eq. (5.4) is a symmetric and positive definite linear system of equations. Its coefficients are computed by assembling the local contributions of all the diamond cells D. The elementary matrix in $\mathrm{D}$ is

$$
\mathbb{K}_{\mathrm{D}}=\left(\begin{array}{lll}
\mathbf{K}_{\mathrm{D}} N_{\mathrm{KL}} \cdot N_{\mathrm{KL}} & \mathbf{K}_{\mathrm{D}} N_{\mathrm{KL}} \cdot N_{\mathrm{AB}} & \mathbf{K}_{\mathrm{D}} N_{\mathrm{KL}} \cdot N_{\mathrm{EF}} \\
\mathbf{K}_{\mathrm{D}} N_{\mathrm{AB}} \cdot N_{\mathrm{KL}} & \mathbf{K}_{\mathrm{D}} N_{\mathrm{AB}} \cdot N_{\mathrm{AB}} & \mathbf{K}_{\mathrm{D}} N_{\mathrm{AB}} \cdot N_{\mathrm{EF}} \\
\mathbf{K}_{\mathrm{D}} N_{\mathrm{EF}} \cdot N_{\mathrm{KL}} & \mathbf{K}_{\mathrm{D}} N_{\mathrm{EF}} \cdot N_{\mathrm{AB}} & \mathbf{K}_{\mathrm{D}} N_{\mathrm{EF}} \cdot N_{\mathrm{EF}}
\end{array}\right)
$$

with $\mathbf{K}_{\mathrm{D}}=\frac{1}{|\mathrm{D}|} \int_{\mathrm{D}} \mathbf{K}(x) d x$. The right hand side in eq. (5.4) is split similarly in elementary contributions on the eight tetrahedra that compose the diamond cells D thanks to $(2.2),(2.3)$ and (2.4).

6. Analysis of the scheme. In this section, the nonlinear system of equations (5.4) is proved to be well-posed, uniform a priori estimates are found on its solutions and finally error estimates are given. The discrete solution $u^{\mathcal{T}}$ will be compared to the pointwise projection of the exact solution $u$, defined as in eq. (5.3) by

$$
\forall u \in W^{2, p}(\Omega), \quad \mathbf{p}^{\mathcal{T}} u=\left(u\left(x_{\mathrm{K}}\right), u\left(x_{\mathrm{A}}\right), u\left(x_{\mathrm{E}}\right), u\left(x_{\mathrm{F}}\right)\right)_{\mathrm{K} \in \mathcal{M}, \mathrm{A} \in \mathcal{N}, \mathrm{E} \in \mathcal{E}, \mathrm{F} \in \mathcal{F}} .
$$

The comparison $u^{\mathcal{T}}-\mathbf{p}^{\mathcal{T}} u$ will be studied in the $L^{p}$ norm as defined by eq. (3.3) and in the discrete $W^{1, p}$ semi norm, defined by

$$
\left|u^{\mathcal{T}}\right|_{W^{1, p}(\Omega), \mathcal{T}}=\left\|\nabla_{0}^{\mathcal{T}} u^{\mathcal{T}}\right\|_{L^{p}(\Omega)} .
$$

6.1. The discrete problem and a priori estimate. First, the discrete problem is proved to be well-posed and the discrete solution $u^{\mathcal{T}}$ to be bounded in the discrete $W^{1, p}$ norm.

Theorem 6.1 (Well-posedness of Eqs. (5.4)). Assume that the flux $\varphi$ satisfies assumptions (1.2), (1.3) and (1.4). For any function $f \in L^{p^{\prime}}(\Omega)$, any boundary data $g=\gamma_{0}(\bar{g})$ with $\bar{g} \in W^{2, p}(\Omega)$ and any mesh $\mathcal{T}$ on $\Omega$, the finite volume scheme (5.4) admits a unique solution $u^{\mathcal{T}} \in X$ and there exists a constant $C>0$ depending only on $p,\left(c_{i}\right)_{i=1,2}$ and $c_{r}(\mathcal{T})$, such that

$$
\int_{\Omega}\left|\nabla_{\delta u}^{\mathcal{T}} u^{\mathcal{T}}\right|^{p} \leq C\left(\|f\|_{L^{p^{\prime}}}^{p^{\prime}}+\left\|b_{1}\right\|_{L^{1}}+\left\|b_{2}\right\|_{L^{p^{\prime}}}^{p^{\prime}}+\|\bar{g}\|_{W^{2, p}(\Omega)}^{p}\right) .
$$


Proof. The mapping $a_{\mathcal{T}}:\left(u^{\mathcal{T}}, \delta u^{\mathcal{T}}\right) \in X \times \delta X \mapsto-\operatorname{div}^{\mathcal{T}}\left(\varphi^{\mathcal{D}}\left(\nabla_{\delta u}^{\mathcal{T}} u^{\mathcal{T}}\right)\right)-\pi^{\mathcal{T}} f \in X$ is continuous and coercitive: its continuity follows from eq. (1.5) and its coercitivity is a consequence of theorems 4.1 and 4.2 and of assumption (1.3).

For any piecewise constant function $v^{\mathcal{T}} \in X$ and piecewise constant boundary data $\delta u^{\mathcal{T}} \in \delta X$, note that $\nabla_{\delta u}^{\mathcal{T}} u^{\mathcal{T}}-\nabla_{\delta u}^{\mathcal{T}} v^{\mathcal{T}}=\nabla_{0}^{\mathcal{T}}\left(u^{\mathcal{T}}-v^{\mathcal{T}}\right)$ so that

$$
\begin{aligned}
\left(a_{\mathcal{T}}\left(u^{\mathcal{T}}, \delta u^{\mathcal{T}}\right), u^{\mathcal{T}}-v^{\mathcal{T}}\right)_{X}=\left(-\operatorname{div}^{\mathcal{T}}\left(\varphi^{\mathcal{D}}\left(\nabla_{\delta u}^{\mathcal{T}} u^{\mathcal{T}}\right)\right)-\pi^{\mathcal{T}} f, u^{\mathcal{T}}-v^{\mathcal{T}}\right)_{X} \\
=\int_{\Omega} \varphi\left(z, \nabla_{\delta u}^{\mathcal{T}} u^{\mathcal{T}}(z)\right) \cdot \nabla_{0}^{\mathcal{T}}\left(u^{\mathcal{T}}(z)-v^{\mathcal{T}}(z)\right) d z-\left(\pi^{\mathcal{T}} f, u^{\mathcal{T}}-v^{\mathcal{T}}\right)_{X} \\
=\int_{\Omega} \varphi\left(z, \nabla_{\delta u}^{\mathcal{T}} u^{\mathcal{T}}(z)\right) \cdot \nabla_{\delta u}^{\mathcal{T}} u^{\mathcal{T}}(z) d z-\int_{\Omega} \varphi\left(z, \nabla_{\delta u}^{\mathcal{T}} u^{\mathcal{T}}(z)\right) \cdot \nabla_{\delta u}^{\mathcal{T}} v^{\mathcal{T}}(z) d z \\
-\left(\pi^{\mathcal{T}} f, u^{\mathcal{T}}-v^{\mathcal{T}}\right)_{X} .
\end{aligned}
$$

Assumptions (1.3) and (1.4) are used to bound the first two terms of the above righthand side:

$$
\int_{\Omega} \varphi\left(z, \nabla_{\delta u}^{\mathcal{T}} u^{\mathcal{T}}(z)\right) \cdot \nabla_{\delta u}^{\mathcal{T}} u^{\mathcal{T}}(z) d z \geq c_{1}\left\|\nabla_{\delta u}^{\mathcal{T}} u^{\mathcal{T}}\right\|_{L^{p}}^{p}-\left\|b_{1}\right\|_{L^{1}}
$$

and

$$
\begin{aligned}
\left|\int_{\Omega} \varphi\left(z, \nabla_{\delta u}^{\mathcal{T}} u^{\mathcal{T}}(z)\right) \cdot \nabla_{\delta u}^{\mathcal{T}} v^{\mathcal{T}}(z) d z\right| & \leq \int_{\Omega}\left(c_{2}\left|\nabla_{\delta u}^{\mathcal{T}} v^{\mathcal{T}}\right|^{p-1}+b_{2}\right)\left|\nabla_{\delta u}^{\mathcal{T}} v^{\mathcal{T}}\right| \\
\leq & c_{2}\left\|\nabla_{\delta u}^{\mathcal{T}} u^{\mathcal{T}}\right\|_{L^{p}}^{p-1}\left\|\nabla_{\delta u}^{\mathcal{T}} v^{\mathcal{T}}\right\|_{L^{p}}+\left\|b_{2}\right\|_{L^{p^{\prime}}}\left\|\nabla_{\delta u}^{\mathcal{T}} v^{\mathcal{T}}\right\|_{L^{p}} .
\end{aligned}
$$

With some inequalities of Young, we get, for any $\alpha, \beta>0$,

$$
\begin{gathered}
\left(a_{\mathcal{T}}\left(u^{\mathcal{T}}, \delta u^{\mathcal{T}}\right), u^{\mathcal{T}}-v^{\mathcal{T}}\right)_{X} \geq\left(c_{1}-\frac{c_{2}}{\beta^{p^{\prime}} p^{\prime}}\right)\left\|\nabla_{\delta u}^{\mathcal{T}} u^{\mathcal{T}}\right\|_{L^{p}}^{p} \\
-\left(\left\|b_{1}\right\|_{L^{1}}+\frac{1}{p^{\prime}}\left\|b_{2}\right\|_{L^{p^{\prime}}}^{p^{\prime}}+\frac{1}{\alpha^{p^{\prime}} p^{\prime}}\left\|\pi^{\mathcal{T}} f\right\|_{L^{p^{\prime}}}^{p^{\prime}}\right)-\left(\frac{c_{2} \beta^{p}}{p}+\frac{1}{p}\right)\left\|\nabla_{\delta u}^{\mathcal{T}} v^{\mathcal{T}}\right\|_{L^{p}}^{p} \\
-\frac{\alpha^{p}}{p}\left\|u^{\mathcal{T}}-v^{\mathcal{T}}\right\|_{L^{p}}^{p} .
\end{gathered}
$$

The inequality of Poincaré (theorem 4.2) is used to bound $\left\|u^{\mathcal{T}}-v^{\mathcal{T}}\right\|_{L^{p}}^{p}$ :

$$
\left\|u^{\mathcal{T}}-v^{\mathcal{T}}\right\|_{L^{p}}^{p} \leq C\left\|\nabla_{0}^{\mathcal{T}}\left(u^{\mathcal{T}}-v^{\mathcal{T}}\right)\right\|_{L^{p}}^{p} \leq C\left\|\nabla_{\delta u}^{\mathcal{T}} u^{\mathcal{T}}\right\|_{L^{p}}^{p}+C\left\|\nabla_{\delta u}^{\mathcal{T}} v^{\mathcal{T}}\right\|_{L^{p}}^{p} .
$$

At last, we can choose $\beta>0$ such that $c_{1}-\frac{c_{2}}{\beta^{p^{\prime}} p^{\prime}}=\frac{1}{2} c_{1}$ and then $\alpha>0$ such that $\frac{1}{2} c_{1}-\frac{C \alpha^{p}}{p}=\frac{1}{4} c_{1}\left(C\right.$ is still the Poincari $; \frac{1}{2}$ constant $)$ and get, because $\left\|\pi^{\mathcal{T}} f\right\|_{L^{p^{\prime}}} \leq$ $\|f\|_{L^{p^{\prime}}}$

$$
\begin{aligned}
\left(a_{\mathcal{T}}\left(u^{\mathcal{T}}, \delta u^{\mathcal{T}}\right), u^{\mathcal{T}}-v^{\mathcal{T}}\right)_{X}+\left(\left\|b_{1}\right\|_{L^{1}}+\frac{1}{p^{\prime}}\left\|b_{2}\right\|_{L^{p^{\prime}}}^{p^{\prime}}+\frac{1}{\alpha^{p^{\prime} p^{\prime}}}\|f\|_{L^{p^{\prime}}}^{p^{\prime}}\right) \\
+\left(\frac{c_{2} \beta^{p}}{p}+\frac{1}{p}+\frac{C \alpha^{p}}{p}\right)\left\|\nabla_{\delta u}^{\mathcal{T}} v^{\mathcal{T}}\right\|_{L^{p}}^{p} \geq \frac{1}{4} c_{1}\left\|\nabla_{\delta u}^{\mathcal{T}} u^{\mathcal{T}}\right\|_{L^{p}}^{p} .
\end{aligned}
$$

Consider $\bar{g} \in W^{2, p}(\Omega)$ such that the boundary data is $g=\gamma_{0}(\bar{g})$. Substituting $v^{\mathcal{T}}=$ $\mathbf{p}^{\mathcal{T}} \bar{g} \in X$ and $\delta u^{\mathcal{T}}=\mathbf{p}^{\mathcal{T}} g \in \delta X$ as defined by eqs. (6.1) and (5.3), lemma 6.8 can be applied and results in

$$
\left\|\nabla_{\delta u}^{\mathcal{T}} v^{\mathcal{T}}\right\|_{L^{p}} \leq C\|\bar{g}\|_{W^{2, p}(\Omega)}
$$


The existence of a solution to the scheme (5.4) is then a consequence of the Brouwer fixed point theorem, and estimate (6.3) is a direct consequence of inequality (6.4).

The uniqueness of the solution is finally due to the monotonicity of the map $a_{\mathcal{T}}$. Indeed, theorem 4.1 and assumption (1.2) yield, for any $u^{\mathcal{T}}, v^{\mathcal{T}} \in X$ such that $u^{\mathcal{T}} \neq v^{\mathcal{T}}$ (with the same boundary data $\delta u^{\mathcal{T}}$ ),

$$
\begin{aligned}
\left(a_{\mathcal{T}}\left(u^{\mathcal{T}}\right)-a_{\mathcal{T}}\left(v^{\mathcal{T}}\right), u^{\mathcal{T}}-v^{\mathcal{T}}\right)_{X} & =\left(-\operatorname{div}^{\mathcal{T}}\left(\varphi^{\mathcal{D}}\left(\nabla_{\delta u}^{\mathcal{T}} u^{\mathcal{T}}\right)\right)+\operatorname{div}^{\mathcal{T}}\left(\varphi^{\mathcal{D}}\left(\nabla_{\delta u}^{\mathcal{T}} v^{\mathcal{T}}\right)\right), u^{\mathcal{T}}-v^{\mathcal{T}}\right)_{X} \\
= & \int_{\Omega}\left(\varphi\left(z, \nabla_{\delta u}^{\mathcal{T}} u^{\mathcal{T}}\right)-\varphi\left(z, \nabla_{\delta u}^{\mathcal{T}} v^{\mathcal{T}}\right)\right) \cdot\left(\nabla_{0}^{\mathcal{T}}\left(u^{\mathcal{T}}-v^{\mathcal{T}}\right)\right) d z \\
& =\int_{\Omega}\left(\varphi\left(z, \nabla_{\delta u}^{\mathcal{T}} u^{\mathcal{T}}\right)-\varphi\left(z, \nabla_{\delta u}^{\mathcal{T}} v^{\mathcal{T}}\right)\right) \cdot\left(\nabla_{\delta u}^{\mathcal{T}} u^{\mathcal{T}}-\nabla_{\delta u}^{\mathcal{T}} v^{\mathcal{T}}\right) d z>0 .
\end{aligned}
$$

Remark: In the case where the flux $\varphi$ derives from a convex potential $\Phi$ :

$$
\varphi(z, \xi)=\nabla_{\xi} \Phi(z, \xi) \forall \xi \in \mathbb{R}^{2} \text {, for a.e. } z \in \Omega \text {, and } \Phi(z, 0)=0 \text { for a.e. } z \in \Omega
$$

the solution $u^{\mathcal{T}}$ of the scheme (5.4) is also the unique minimizer of the discrete energy $J^{\mathcal{T}}$ associated to the scheme by $J^{\mathcal{T}}\left(u^{\mathcal{T}}\right)=\int_{\Omega} \Phi\left(z, \nabla^{\mathcal{T}} u^{\mathcal{T}}\right)-\int_{\Omega} u^{\mathcal{T}} \pi^{\mathcal{T}} f$.

6.2. Assumptions and technical lemmas. The scheme is well-posed, and the discrete solution might be proved to converge under assumptions (1.2), (1.3) and (1.4) only, following the 2D case [3] or another 3D approach [2]. Anyway, in order to compute some error estimates, the following additional assumptions on $\varphi$ are needed : there exists constants $c_{3}, c_{4}, c_{5}>0, b_{3} \in L^{\frac{p}{p-2}}(\Omega)$ and a function $b_{4} \in L^{p^{\prime}}(\Omega)$ such that for all $(\xi, \eta) \in \mathbb{R}^{3} \times \mathbb{R}^{3}$ and almost every $z \in \Omega$,

$$
\begin{gathered}
(\varphi(z, \xi)-\varphi(z, \eta)) \cdot(\xi-\eta) \geq c_{3}|\xi-\eta|^{p} \\
|\varphi(z, \xi)-\varphi(z, \eta)| \leq c_{4}\left(b_{3}(z)+|\xi|^{p-2}+|\eta|^{p-2}\right)|\xi-\eta|,
\end{gathered}
$$

and for all $\xi \in \mathbb{R}^{3}$ and almost every $z \in \Omega$,

$$
\left|\frac{\partial \varphi}{\partial z}(z, \xi)\right| \leq c_{5}\left(b_{4}(z)+|\xi|^{p-1}\right) .
$$

For $p=2$, inequality (6.6) degenerates and the assumptions becomes $b_{3} \in L^{\infty}(\Omega)$.

Lemma 6.2 (Regularity of the flux). Under assumptions (6.5)-(6.7) on $\varphi$ and given a function $u \in W^{2, p}(\Omega)$, the mapping $G: z \mapsto \varphi(z, \nabla v(z))$ is well defined in $\left(W^{1, p^{\prime}}(\Omega)\right)^{3}$. Furthermore, the mapping $v \mapsto G$ is such that

$$
\|\nabla G\|_{L^{p^{\prime}}(\Omega)} \leq C\|v\|_{W^{2, p}(\Omega)}
$$

with $C>0$ that depends only on the $\left(b_{i}\right)_{i=3,4}$ and $\left(c_{i}\right)_{i=3,4,5}$.

Proof. The proof is straightforward.

Definition 6.3 (Errors of consistency). Suppose that the solution $u$ of the continuous problem (1.1) is in $W^{2, p}(\Omega)$. Consider its pointwise projection $\left(\mathbf{p}^{\mathcal{T}} u, \mathbf{p}^{\mathcal{T}} g\right) \in$ $X \times \delta X$ defined by eqs (6.1) and (5.3). The approximate flux associated to the projection $\left(\mathbf{p}^{\mathcal{T}} u, \mathbf{p}^{\mathcal{T}} g\right)$ is exactly $\varphi^{\mathcal{D}}\left(\nabla_{\mathbf{p}_{g}}^{\mathcal{T}} \mathbf{p}^{\mathcal{T}} u\right) \in \mathbf{Q}$ while there are two discrete flux 
functions associated to the function $u$, namely the piecewise constant projection of $\varphi(x, \nabla u(x))$ onto the diamond cells, denoted by $\varphi^{\mathcal{D}}(\nabla u)=\left(\varphi_{\mathrm{D}}(\nabla u)\right)_{\mathrm{D} \in \mathcal{D}} \in \mathbf{Q}$ with

$$
\varphi_{\mathrm{D}}(\nabla u)=\frac{1}{|\mathrm{D}|} \int_{\mathrm{D}} \varphi(x, \nabla u(x)) d x
$$

and the function $\psi_{\mathcal{T}}(\nabla u)=\left(\psi_{\mathrm{D}}(\nabla u)\right)_{\mathrm{D} \in \mathcal{D}} \in \mathbf{Q}$ where the vectors $\psi_{\mathrm{D}}(\nabla u) \in \mathbb{R}^{3}$ are defined uniquely for any diamond cell $\mathrm{D} \in \mathcal{D}$ by the three equations

$$
\begin{aligned}
& \psi_{\mathrm{D}}(\nabla u) \cdot N_{\mathrm{KL}}=F_{\mathrm{KL}}^{\mathrm{D}}:=\int_{S_{\mathrm{KL}}} \varphi(z, \nabla u(z)) \cdot n_{\mathrm{KL}} d z, \\
& \psi_{\mathrm{D}}(\nabla u) \cdot N_{\mathrm{AB}}=F_{\mathrm{AB}}^{\mathrm{D}}:=\int_{S_{\mathrm{AB}}} \varphi(z, \nabla u(z)) \cdot n_{\mathrm{AB}} d z, \\
& \psi_{\mathrm{D}}(\nabla u) \cdot N_{\mathrm{EF}}=F_{\mathrm{EF}}^{\mathrm{D}}:=\int_{S_{\mathrm{EF}}} \varphi(z, \nabla u(z)) \cdot n_{\mathrm{EF}} d z
\end{aligned}
$$

(i.e. the numbers $F^{\mathrm{D}}$ are the exact fluxes through the 3 interfaces inside the diamond cell $\mathrm{D})$. The total error of consistency is $R_{\mathcal{T}}(u)=\left(R_{\mathrm{D}}(u)\right)_{\mathrm{D} \in \mathcal{D}} \in \mathbf{Q}$ where

$$
\forall \mathrm{D} \in \mathcal{D}, \quad R_{\mathrm{D}}(u)=\psi_{\mathrm{D}}(\nabla u)-\varphi_{\mathrm{D}}\left(\nabla_{\mathbf{p}_{g}}^{\mathcal{T}} \mathbf{p}^{\mathcal{T}} u\right) .
$$

It is split as $R_{\mathcal{T}}(u)=R_{\mathcal{T}}^{1}(u)+R_{\mathcal{T}}^{2}(u)$ with $R_{\mathcal{T}}^{j}(u)=\left(R_{\mathrm{D}}^{j}(u)\right)_{\mathrm{D} \in \mathcal{D}}$ and

$$
\forall \mathrm{D} \in \mathcal{D}, \quad R_{\mathrm{D}}^{1}(u)=\psi_{\mathrm{D}}(\nabla u)-\varphi_{\mathrm{D}}(\nabla u)
$$

involves differences between surface and volume integrals and

$$
\forall \mathrm{D} \in \mathcal{D}, \quad R_{\mathrm{D}}^{2}(u)=\varphi_{\mathrm{D}}(\nabla u)-\varphi_{\mathrm{D}}\left(\nabla_{\mathbf{p}_{g}}^{\mathcal{T}} \mathbf{p}^{\mathcal{T}} u\right)
$$

is related to the error of consistency on the gradient.

The next lemma are technical multidimensional results used to bound the errors term $R_{\mathrm{D}}^{1}$ and $R_{\mathrm{D}}^{2}$, similar to the usual finite element estimates, see for instance [6].

Lemma 6.4. Let $H=\left\{x,\left(x-x_{0}\right) \cdot n=0\right\}\left(x_{0} \in \mathbb{R}^{d}\right.$ and $\left.n \in \mathbb{R}^{d}\right)$ be a hyperplane in $\mathbb{R}^{d}$ and $\sigma \subset H$ be an bounded polygon in $H$. Consider $x_{1}$ and $x_{2}$ in $\mathbb{R}^{d} \backslash H$ such that $\left(x_{1}-x_{0}\right) \cdot n<0<\left(x_{2}-x_{0}\right) \cdot n$ and let $K_{i}(i=1,2)$ be the pyramid with basis $\sigma$ and vertex $x_{i}$, namely $K_{i}=\left\{t x_{i}+(1-t) y, y \in \sigma, t \in(0,1)\right\}$. Finally consider the set $K=K_{1} \cup K_{2}$. For any $q \geq 1$ and any function $v \in W^{1, q}(K)$,

$$
\begin{aligned}
&\left|\frac{1}{\left|K_{i}\right|} \int_{K_{i}} v(x) d x-\frac{1}{|\sigma|} \int_{\sigma} v(y) d \sigma(y)\right| \leq \frac{h_{i}}{d\left|K_{i}\right|^{1 / q}}\|\nabla v\|_{L^{q}\left(K_{i}\right)} \quad(i=1,2), \\
&\left|\frac{1}{\left|K_{1}\right|} \int_{K_{1}} v(x) d x-\frac{1}{\left|K_{2}\right|} \int_{K_{2}} v(x) d x\right| \leq \frac{1}{d}\left(\max _{i=1,2} \frac{h_{i}}{\left|K_{i}\right|}\right)|K|^{1-1 / q}\|\nabla v\|_{L^{q}(K)},
\end{aligned}
$$

where $h_{i}=\operatorname{diam}\left(K_{i}\right)=\max \{|x-y|, x, y \in \bar{K}\}$.

Proof. The first inequality is a variant of the result proved in [12] (lemma 6.3) while the second is an easy consequence of the former one. $\square$

Lemma 6.5. Consider an open bounded subset $K$ in $\mathbb{R}^{d}$ and suppose that there exists an open ball $B$ of center $x_{K} \in K$ and of radius $R>0$ such that $K$ is star-shaped with respect to $B$. For any $q \geq 1$ and for any function $v \in W^{1, q}(K)$,

$$
\left\|v-v_{B}\right\|_{L^{q}(K)} \leq\left(\frac{d}{d-1}\right)^{1 / q} \frac{h_{K}^{2}}{R}\|\nabla v\|_{L^{q}(K)}
$$


where $h_{K}=\sup \{|x-y|, x, y \in K\}$ is the diameter of $K$ and $v_{B}=\frac{1}{|B|} \int_{B} v(x) d x$ is the average value of $v$ on $B$.

Proof. The proof is an adaptation of the proof of lemma 7.1 in [11]. $\square$

LEMMA 6.6 (Error of consistency due to the projections). If $u \in W^{2, p}(\Omega)$ then

$$
\left\|R_{\mathcal{T}}^{1}(u)\right\|_{L^{p^{\prime}}(\Omega)} \leq 2 c_{r}(\mathcal{T})^{2} h\|\nabla G\|_{L^{p^{\prime}}(\Omega)} \leq 2 C c_{r}(\mathcal{T})^{2} h\|u\|_{W^{2, p}(\Omega)},
$$

where the constant $C>0$ and the function $G$ are taken from lemma 6.2.

Proof. The difference $R_{\mathrm{D}}^{1}(u)$ involves differences between surface and volume averages of the flux:

$$
\begin{aligned}
\left|R_{\mathrm{D}}^{1}(u) \cdot \nu_{\mathrm{KL}}\right| & =\left|\frac{1}{\left|N_{\mathrm{KL}}\right|} \int_{S_{\mathrm{KL}}} \varphi(z, \nabla u(z)) \cdot n_{\mathrm{KL}} d z-\frac{1}{|\mathrm{D}|} \int_{\mathrm{D}} \varphi(z, \nabla u(z)) \cdot \nu_{\mathrm{KL}} d z\right|, \\
\left|R_{\mathrm{D}}^{1}(u) \cdot \nu_{\mathrm{AB}}\right| & =\left|\frac{1}{\left|N_{\mathrm{AB}}\right|} \int_{S_{\mathrm{AB}}} \varphi(z, \nabla u(z)) \cdot n_{\mathrm{AB}} d z-\frac{1}{|\mathrm{D}|} \int_{\mathrm{D}} \varphi(z, \nabla u(z)) \cdot \nu_{\mathrm{AB}} d z\right|, \\
\left|R_{\mathrm{D}}^{1}(u) \cdot \nu_{\mathrm{EF}}\right| & =\left|\frac{1}{\left|N_{\mathrm{EF}}\right|} \int_{S_{\mathrm{EF}}} \varphi(z, \nabla u(z)) \cdot n_{\mathrm{EF}} d z-\frac{1}{|\mathrm{D}|} \int_{\mathrm{D}} \varphi(z, \nabla u(z)) \cdot \nu_{\mathrm{EF}} d z\right| .
\end{aligned}
$$

Remark that each of the interfaces $S_{\mathrm{KL}}, S_{\mathrm{AB}}$ and $S_{\mathrm{EF}}$ is composed of four triangles (eqs. (2.5), (2.7) and (2.9) from lemma 2.2), while D is a collection of 8 tetrahedra with theses faces as bases and, respectively, $\left(x_{\mathrm{K}}, x_{\mathrm{L}}\right),\left(x_{\mathrm{A}}, x_{\mathrm{B}}\right)$ and $\left(x_{\mathrm{E}}, x_{\mathrm{F}}\right)$ as vertices.

For sake of simplicity, let $S$ denote any of these interfaces, $S_{i}$ denote the four triangles $(i=1 \ldots 4), x_{1}, x_{2}$ denote the two corresponding vertices and $n(x)=$ $\sum_{i=1}^{4} n_{i} \chi_{S_{i}}(x)$ denote the unit normal to $S$ from $x_{1}$ towards $x_{2}$. The diamond cell is composed of the 8 tetrahedra $\mathrm{D}_{i}^{k}=\operatorname{hull}\left(S_{i}, x_{k}\right)(i=1, \ldots 4$ and $k=1,2)$. The corresponding integral normal is $N=\int_{S} n(x) d \sigma(x)=\sum_{i=1}^{4}\left|S_{i}\right| n_{i}$ and the unit normal is $\nu=\sum_{i=1}^{4} \frac{\left|S_{i}\right|}{|S|} n_{i}$. At last, consider the function $G(z)=\varphi(z, \nabla u(z))$ as defined in lemme 6.2. Each of the 3 errors from above now reads

$$
\begin{aligned}
R_{\mathrm{D}}^{1}(u) \cdot \nu & =\sum_{i=1}^{4} \frac{\left|S_{i}\right|}{|S|} n_{i} \cdot\left(\frac{1}{\left|S_{i}\right|} \int_{S_{i}} G(z) d z-\frac{1}{|\mathrm{D}|} \int_{\mathrm{D}} G(z) d z\right) \\
& =\sum_{j=1}^{4} \sum_{i=1}^{4} \sum_{k=1,2} \frac{\left|\mathrm{D}_{j}^{k}\right|}{|\mathrm{D}|} \frac{\left|S_{i}\right|}{|S|} n_{i} \cdot\left(\frac{1}{\left|S_{i}\right|} \int_{S_{i}} G(z) d z-\frac{1}{\left|\mathrm{D}_{j}^{k}\right|} \int_{\mathrm{D}_{j}^{k}} G(z) d z\right)
\end{aligned}
$$

and it remains to bound $\left|\frac{1}{\left|S_{i}\right|} \int_{S_{i}} G(z) d z-\frac{1}{\left|\mathrm{D}_{j}^{k}\right|} \int_{\mathrm{D}_{j}^{k}} G(z) d z\right|$ for any $i=1 \ldots 4, j=$ $1 \ldots 4$ and $k=1,2$. Since the $\mathrm{D}_{j}^{k}$ form a tetrahedrisation of $\mathrm{D}$ the following bound is a consequence of lemma 6.4 with $q=1$ and $d=3$ :

$$
\begin{aligned}
& \left|\frac{1}{\left|S_{i}\right|} \int_{S_{i}} G(z) d z-\frac{1}{\left|\mathrm{D}_{j}^{k}\right|} \int_{\mathrm{D}_{j}^{k}} G(z) d z\right| \\
& \leq\left|\frac{1}{\left|S_{i}\right|} \int_{S_{i}} G(z) d z-\frac{1}{\left|\mathrm{D}_{i}^{k}\right|} \int_{\mathrm{D}_{i}^{k}} G(z) d z\right|+\left|\frac{1}{\left|\mathrm{D}_{i}^{k}\right|} \int_{\mathrm{D}_{i}^{k}} G(z) d z-\frac{1}{\left|\mathrm{D}_{j}^{k}\right|} \int_{\mathrm{D}_{j}^{k}} G(z) d z\right| \\
& \leq \frac{h_{i, k}}{3\left|\mathrm{D}_{i}^{k}\right|} \int_{\mathrm{D}_{i}^{k}}|\nabla G(z)| d z+\frac{1}{3}\left(\max _{j=1, \ldots 4} \frac{h_{j, k}}{\left|\mathrm{D}_{j}^{k}\right|}\right) \int_{\mathrm{D}^{k}}|\nabla G(z)| d z \\
& \leq \frac{2}{3}\left(\max _{j=1, \ldots 4} \frac{h_{j, k}}{\left|\mathrm{D}_{j}^{k}\right|}\right) \int_{\mathrm{D}^{k}}|\nabla G(z)| d z
\end{aligned}
$$


where $\mathrm{D}^{k}=\cup_{j=1}^{4} \mathrm{D}_{j}^{k}$ and $h_{j, k}=\operatorname{diam}\left(\mathrm{D}_{j}^{k}\right)$. Finally, since $\mathrm{D}^{k} \subset \mathrm{D}$,

$$
\begin{aligned}
\left|R_{\mathrm{D}}^{1}(u) \cdot \nu\right| & \leq \frac{2}{3}\left(\max _{k=1,2} \max _{j=1, \ldots 4} \frac{h_{j, k}}{\left|\mathrm{D}_{j}^{k}\right|}\right) \int_{\mathrm{D}}|\nabla G(z)| d z \sum_{j=1}^{4} \sum_{i=1}^{4} \sum_{k=1,2} \frac{\left|\mathrm{D}_{j}^{k}\right|}{|\mathrm{D}|} \frac{\left|S_{i}\right|}{|S|} \\
& \leq \frac{2}{3}\left(\max _{k=1,2} \max _{j=1, \ldots 4} \frac{h_{j, k}}{\left|\mathrm{D}_{j}^{k}\right|}\right)|\mathrm{D}|^{1 / p}\|\nabla G\|_{L^{p^{\prime}}(\mathrm{D})} \\
& \leq \frac{2 c_{r}(\mathcal{T})}{3} h|\mathrm{D}|^{-1 / p^{\prime}}\|\nabla G\|_{L^{p^{\prime}}(\mathrm{D})}
\end{aligned}
$$

because $c_{r}(\mathcal{T})\left|\mathrm{D}_{j}^{k}\right| \geq|\mathrm{D}|$ and $h_{j, k} \leq h_{\mathrm{D}} \leq h$. The vector $R_{\mathrm{D}}^{1}(u)$ can be expressed in the basis $\left(\nu_{\mathrm{KL}}, \nu_{\mathrm{AB}}, \nu_{\mathrm{EF}}\right)$ as $R_{\mathrm{D}}^{1}(u)=R_{\mathrm{KL}}^{1}(u) \nu_{\mathrm{KL}}+R_{\mathrm{AB}}^{1}(u) \nu_{\mathrm{AB}}+R_{\mathrm{EF}}^{1}(u) \nu_{\mathrm{EF}}$ and its norm verifies

$$
\left|R_{\mathrm{D}}^{1}(u)\right|^{2}=R_{\mathrm{D}}^{1}(u)^{T} G_{\mathrm{D}} R_{\mathrm{D}}^{1}(u) \leq \lambda_{M}(\mathrm{D})\left(\left(R_{\mathrm{KL}}^{1}(u)\right)^{2}+\left(R_{\mathrm{AB}}^{1}(u)\right)^{2}+\left(R_{\mathrm{EF}}^{1}(u)\right)^{2}\right) .
$$

The three errors of consistency evaluated above are $R_{\mathrm{D}}^{1}(u) \cdot \nu_{\mathrm{KL}}, \ldots$ given by

$$
\left(\begin{array}{l}
R_{\mathrm{D}}^{1} \cdot \nu_{\mathrm{KL}} \\
R_{\mathrm{D}}^{1} \cdot \nu_{\mathrm{AB}} \\
R_{\mathrm{D}}^{1} \cdot \nu_{\mathrm{EF}}
\end{array}\right)=G_{\mathrm{D}}\left(\begin{array}{l}
R_{\mathrm{KL}}^{1} \\
R_{\mathrm{AB}}^{1} \\
R_{\mathrm{EF}}^{1}
\end{array}\right) .
$$

Because $\left\|G_{\mathrm{D}}^{-1}\right\|_{2} \leq \frac{1}{\lambda_{m}(\mathrm{D})}$, it is derived the estimate

$$
\left|R_{\mathrm{D}}^{1}(u)\right|^{2} \leq \frac{\lambda_{M}(\mathrm{D})}{\lambda_{m}(\mathrm{D})^{2}}\left(\left(R_{\mathrm{D}}^{1}(u) \cdot \nu_{\mathrm{KL}}\right)^{2}+\left(R_{\mathrm{D}}^{1}(u) \cdot \nu_{\mathrm{AB}}\right)^{2}+\left(R_{\mathrm{D}}^{1}(u) \cdot \nu_{\mathrm{EF}}\right)^{2}\right) .
$$

Here, $\lambda_{M}(\mathrm{D})$ is the largest eigenvalue of the Gram matrix $G_{\mathrm{D}}$ as defined by eq. (2.15), while $\lambda_{m}(\mathrm{D})$ is its smallest one. Note that $\lambda_{M}(\mathrm{D}) \leq\left\|G_{\mathrm{D}}\right\|_{1} \leq 3$ and $c_{r}(\mathcal{T}) \lambda_{m}(\mathrm{D}) \geq 1$, so that the bound on the local error of consistency reads

$$
\left|R_{\mathrm{D}}^{1}(u)\right| \leq 3 c_{r}(\mathcal{T}) \frac{2 c_{r}(\mathcal{T})}{3} h|\mathrm{D}|^{-1 / p^{\prime}}\|\nabla G\|_{L^{p^{\prime}}(\mathrm{D})}=2 c_{r}(\mathcal{T})^{2} h|\mathrm{D}|^{-1 / p^{\prime}}\|\nabla G\|_{L^{p^{\prime}}(\mathrm{D})}
$$

and the proof is completed by an easy summation over the diamond cell D in $\mathcal{D}$. $\square$

Lemma 6.7 (Error of consistency on the flux). There exists a constant $C>0$ depending on the mesh regularity constant $c_{r}(\mathcal{T})$, on $c_{4}$ and $p, q$, such that, if $u \in$ $W^{2, p}(\Omega)$,

$$
\left\|R_{\mathcal{T}}^{2}(u)\right\|_{L^{p^{\prime}}(\Omega)} \leq C h\left(\left\|b_{3}\right\|_{L^{p /(p-2)}(\Omega)}+\|u\|_{W^{2, p}(\Omega)}^{p-2}\right)\|u\|_{W^{2, p}(\Omega)}
$$

with $g=\gamma(u)$ as a boundary data. 
Proof. Using (6.6), the computation of $R_{\mathcal{T}}^{2}(u)$ reads for $p \neq 2$ :

$$
\begin{gathered}
\int_{\Omega}\left|R_{\mathcal{T}}^{2}(u)\right|^{p^{\prime}}=\sum_{\mathrm{D} \in \mathcal{D}}|\mathrm{D}|\left|\frac{1}{|\mathrm{D}|} \int_{\mathrm{D}}\left(\varphi(z, \nabla u(z))-\varphi\left(z, \nabla_{\mathbf{p}_{g}}^{\mathrm{D}} \mathbf{p}^{\mathcal{T}} u(z)\right)\right) d z\right|^{p^{\prime}} \\
\leq \sum_{\mathrm{D} \in \mathcal{D}} \int_{\mathrm{D}}\left|\varphi(z, \nabla u(z))-\varphi\left(z, \nabla_{\mathbf{p}_{g}}^{\mathrm{D}} \mathbf{p}^{\mathcal{T}} u(z)\right) d z\right|^{p^{\prime}} \\
\leq \sum_{\mathrm{D} \in \mathcal{D}} \int_{\mathrm{D}}\left|c_{4}\left(b_{3}+|\nabla u|^{p-2}+\left|\nabla_{\mathbf{p}_{g}}^{\mathrm{D}} \mathbf{p}^{\mathcal{T}} u\right|^{p-2}\right)\right|^{p^{\prime}}\left|\nabla u-\nabla_{\mathbf{p}_{g}}^{\mathrm{D}} \mathbf{p}^{\mathcal{T}} u\right|^{p^{\prime}} d z \\
\leq\left(\int_{\Omega}\left|c_{4}\left(b_{3}+|\nabla u|^{p-2}+\left|\nabla_{\mathbf{p}_{g}}^{\mathcal{T}} \mathbf{p}^{\mathcal{T}} u\right|^{p-2}\right)\right|^{p /(p-2)} d z\right)^{1-p^{\prime} / p} \\
\left(\int_{\Omega}\left|\nabla u-\nabla_{\mathbf{p}_{g}}^{\mathcal{T}} \mathbf{p}^{\mathcal{T}} u\right|^{p} d z\right)^{p^{\prime} / p}
\end{gathered}
$$

because $p^{\prime} /\left(1-p^{\prime} / p\right)=1 /(1-2 / p)=p /(p-2)$. This inequality finally reads

$$
\begin{aligned}
&\left\|R_{\mathcal{T}}^{2}(u)\right\|_{L^{p^{\prime}}(\Omega)} \leq c_{4}^{1-2 / p}\left\|b_{3}+|\nabla u|^{p-2}+\left|\nabla_{\mathbf{p}_{g}}^{\mathcal{T}} \mathbf{p}^{\mathcal{T}} u\right|^{p-2}\right\|_{L^{p /(p-2)}}\left\|\nabla u-\nabla_{\mathbf{p}_{g}}^{\mathcal{T}} \mathbf{p}^{\mathcal{T}} u\right\|_{L^{p}(\Omega)} \\
& \leq c_{4}^{1-2 / p}\left(\left\|b_{3}\right\|_{L^{p /(p-2)}(\Omega)}+\|\nabla u\|_{L^{p}(\Omega)}^{p-2}+\left\|\nabla_{\mathbf{p}_{g}}^{\mathcal{T}} \mathbf{p}^{\mathcal{T}} u\right\|_{L^{p}(\Omega)}^{p-2}\right)\left\|\nabla u-\nabla_{\mathbf{p}_{g}}^{\mathcal{T}} \mathbf{p}^{\mathcal{T}} u\right\|_{L^{p}(\Omega)} .
\end{aligned}
$$

For $p=2$, we simply have

$$
\begin{aligned}
\int_{\Omega}\left|R_{\mathcal{T}}^{2}(u)\right|^{2} \leq \sum_{\mathrm{D} \in \mathcal{D}} \int_{\mathrm{D}}\left|\varphi(z, \nabla u(z))-\varphi\left(z, \nabla_{\mathbf{p}_{g}}^{\mathrm{D}} \mathbf{p}^{\mathcal{T}} u(z)\right) d z\right|^{2} & \\
& \leq\left\|b_{3}\right\|_{L^{\infty}(\Omega)}\left\|\nabla u-\nabla_{\mathbf{p}_{g}}^{\mathrm{D}} \mathbf{p}^{\mathcal{T}} u\right\|_{L^{2}(\Omega)}^{2} .
\end{aligned}
$$

The final estimate results from lemma 6.8 below.

Lemma 6.8 (Estimates on the projection). There exists a constant $C(p)>0$ that depends only on $p$ such that, for any function $u \in W^{2, p}(\Omega)$,

$$
\begin{array}{r}
\left\|\nabla u-\nabla_{\mathbf{p}_{g}}^{\mathcal{T}} \mathbf{p}^{\mathcal{T}} u\right\|_{L^{p}(\Omega)} \leq C(p) c_{r}(\mathcal{T})^{3(1+1 / p)} h\|u\|_{W^{2, p}(\Omega)}, \\
\left\|\nabla_{\mathbf{p}_{g}}^{\mathcal{T}} \mathbf{p}^{\mathcal{T}} u\right\|_{L^{p}(\Omega)} \leq\left(1+C(p) c_{r}(\mathcal{T})^{3(1+1 / p)} \operatorname{diam}(\Omega)\right)\|u\|_{W^{2, p}(\Omega)}
\end{array}
$$

with $g=\gamma(u)$. The value of $C(p)$ is specified in eq. (6.15).

Proof. By density, it is sufficient to prove the estimate (6.12) for functions $u \in$ $C^{2}(\bar{\Omega})$. In that case, for any $\mathrm{D} \in \mathcal{D}$, consider the ball $B_{\mathrm{D}}$ inside $\mathrm{D}$ of radius $R_{\mathrm{D}}>0$ and such that $\mathrm{D}$ is star-shaped with respect to $B_{\mathrm{D}}$ (definition 2.3). It is written $\left\|\nabla u-\nabla_{\mathbf{p}_{g}}^{\mathcal{T}} \mathbf{p}^{\mathcal{T}} u\right\|_{L^{p}(\Omega)}=\left(\sum_{\mathrm{D} \in \mathcal{D}} E_{\mathrm{D}}\right)^{1 / p}$ with $E_{\mathrm{D}}=\int_{\mathrm{D}}\left|\nabla u(z)-\nabla_{\mathbf{p}_{g}}^{\mathrm{D}} \mathbf{p}^{\mathcal{T}} u\right|^{p} d z$. Let $\langle\nabla u\rangle_{B}=\frac{1}{|B|} \int_{B} \nabla u(z) d z$ denote the average of $\nabla u$ on the ball $B$. The estimate is split as

$$
E_{\mathrm{D}} \leq 2^{p-1}\left(\int_{\mathrm{D}}\left|\nabla u(z)-\langle\nabla u\rangle_{B}\right|^{p} d z+\left|\langle\nabla u\rangle_{B}-\nabla_{\mathbf{p}_{g}}^{\mathrm{D}} \mathbf{p}^{\mathcal{T}} u\right|^{p}|\mathrm{D}|\right) .
$$

The first term is bounded because of the result from lemma 6.5 ,

$$
\int_{\mathrm{D}}\left|\nabla u(z)-\langle\nabla u\rangle_{B}\right|^{p} d z \leq \frac{3}{2}\left(\frac{h_{\mathrm{D}}}{R_{\mathrm{D}}}\right)^{p} h_{\mathrm{D}}^{p} \int_{\mathrm{D}}|\nabla u(z)|^{p} d z .
$$


To bound the second term, note that, for any $\mathrm{C}=\mathrm{K}, \mathrm{L}, \mathrm{A}, \mathrm{B}, \mathrm{E}, \mathrm{F}$,

$$
\forall z \in B, \quad u\left(x_{\mathrm{C}}\right)=u(z)+\nabla u(z) \cdot\left(x_{\mathrm{C}}-z\right)+E_{\mathrm{C}}(z),
$$

with $E_{\mathrm{C}}(z)=\int_{0}^{1} H(u)\left(z+t\left(x_{\mathrm{C}}-z\right)\right)\left(x_{\mathrm{C}}-z\right) \cdot\left(x_{\mathrm{C}}-z\right)(1-t) d t$ (where $H(u)$ denotes the Hessian matrix of $u$ ). From the consistency property stated in lemma 3.1 and because $g=\gamma_{0}(u)$,

$$
\begin{aligned}
\nabla_{\mathbf{p}_{g}}^{\mathrm{D}} \mathbf{p}^{\mathcal{T}} u=\nabla u(z)+\frac{1}{3|\mathrm{D}|}\left(\left(E_{\mathrm{L}}(z)-E_{\mathrm{K}}(z)\right) N_{\mathrm{KL}}+\left(E_{\mathrm{B}}(z)\right.\right. & \left.-E_{\mathrm{A}}(z)\right) N_{\mathrm{AB}} \\
& \left.+\left(E_{\mathrm{F}}(z)-E_{\mathrm{E}}(z)\right) N_{\mathrm{EF}}\right) .
\end{aligned}
$$

As a consequence,

$$
\nabla_{\mathbf{p}_{g}}^{\mathrm{D}} \mathbf{p}^{\mathcal{T}} u-\langle\nabla u\rangle_{B}=\frac{1}{3|\mathrm{D}|}\left(\left(E_{\mathrm{L}}-E_{\mathrm{K}}\right) N_{\mathrm{KL}}+\left(E_{\mathrm{B}}-E_{\mathrm{A}}\right) N_{\mathrm{AB}}+\left(E_{\mathrm{F}}-E_{\mathrm{E}}\right) N_{\mathrm{EF}}\right)
$$

with $E_{\mathrm{C}}=\frac{1}{|B|} \int_{B} E_{\mathrm{C}}(z) d z$. The estimate of $E_{\mathrm{C}}$ is carried out as usual, with the change of variable $s=1-t$ and because $\left|z-x_{\mathrm{C}}\right| \leq h_{\mathrm{D}}$, and then with the change of variable $x=s z+(1-s) x_{\mathrm{C}}$ :

$$
\left|E_{\mathrm{C}}\right| \leq \frac{h_{\mathrm{D}}^{2}}{|B|} \int_{B} \int_{0}^{1}\left|H(u)\left(s z+(1-s) x_{\mathrm{C}}\right)\right| s d s d z \leq \frac{h_{\mathrm{D}}^{2}}{|B|} \int_{0}^{1} \int_{B(s)}|H(u)(x)| d x \frac{s d s}{s^{d}}
$$

where $d=3$ is the dimension and $B(s)=\left\{s z+(1-s) x_{\mathrm{C}}, z \in B\right\} \subset \mathrm{D}$ because $\mathrm{D}$ is star-shaped with respect to $B$. Due to the inequality of Hölder, $\int_{B(s)}|H(u)(x)| d x \leq$ $\|H(u)\|_{L^{p}(\mathrm{D})}|B(s)|^{1-1 / p}$ with $|B(s)|=s^{d}|B|$ and it finally reads

$$
\begin{aligned}
\left|E_{\mathrm{C}}\right| \leq \frac{h_{\mathrm{D}}^{2}}{|B|}\|H(u)\|_{L^{p}(\mathrm{D})} \int_{0}^{1} s^{d(1-1 / p)}|B|^{1-1 / p} \frac{s d s}{s^{d}} \\
\quad=h_{\mathrm{D}}^{2}|B|^{-1 / p}\|H(u)\|_{L^{p}(\mathrm{D})} \int_{0}^{1} s^{1-d / p} d s=\frac{1}{2-d / p} h_{\mathrm{D}}^{2}|B|^{-1 / p}\|H(u)\|_{L^{p}(\mathrm{D})} .
\end{aligned}
$$

Here, $2-d / p>0$ because $d=3$ and $p \geq 2$, which is also used to prove the Sobolev's embeddings stated in (1.6). The final estimate of the second term of eq. (6.14) is

$$
\begin{array}{r}
\left|\langle\nabla u\rangle_{B}-\nabla_{\mathbf{p}_{g}}^{\mathrm{D}} \mathbf{p}^{\mathcal{T}} u\right| \leq \frac{1}{3|\mathrm{D}|} \frac{2}{2-3 / p} h_{\mathrm{D}}^{2}|B|^{-1 / p}\|H(u)\|_{L^{p}(\mathrm{D})}\left(\left|N_{\mathrm{KL}}\right|+\left|N_{\mathrm{AB}}\right|+\left|N_{\mathrm{EF}}\right|\right) \\
\leq \frac{1}{3|\mathrm{D}|} \frac{2}{2-3 / p} h_{\mathrm{D}}^{2}|B|^{-1 / p}\|H(u)\|_{L^{p}(\mathrm{D})} \frac{3}{2} h_{\mathrm{D}}^{2}
\end{array}
$$

because $\left|N_{\mathrm{KL}}\right|,\left|N_{\mathrm{AB}}\right|$ and $\left|N_{\mathrm{EF}}\right| \leq \frac{1}{2} h_{\mathrm{D}}^{2}$ in view of eqs. (2.6), (2.8) and (2.10). At last, the bound on each $E_{\mathrm{D}}$ reads

$$
E_{\mathrm{D}} \leq 2^{p-1}\left(\frac{3}{2}\left(\frac{h_{\mathrm{D}}}{R_{\mathrm{D}}}\right)^{p} h_{\mathrm{D}}^{p}+\frac{1}{(2-3 / p)^{p}} \frac{h_{\mathrm{D}}^{4 p}}{|\mathrm{D}|^{p}} \frac{|\mathrm{D}|}{|B|}\right)\|u\|_{W^{2, p}(\mathrm{D})}^{p}
$$

and the conclusion holds because $h_{\mathrm{D}} \leq c_{r}(\mathcal{T}) R_{\mathrm{D}}$ and

$$
\frac{4}{3} \pi R_{\mathrm{D}}^{3}=|B| \leq|D| \leq \frac{4}{3} \pi \frac{h_{\mathrm{D}}^{3}}{2^{3}} \text { and then } \frac{h_{\mathrm{D}}^{3}}{|\mathrm{D}|} \leq \frac{3}{4 \pi} \frac{h_{\mathrm{D}}^{3}}{R_{\mathrm{D}}^{3}}, \frac{|\mathrm{D}|}{|B|} \leq \frac{1}{8} \frac{h_{\mathrm{D}}^{3}}{R_{\mathrm{D}}^{3}} .
$$


Specifically, $E_{\mathrm{D}} \leq C(p)^{p} c_{r}(\mathcal{T})^{3(p+1)} h_{\mathrm{D}}^{p}\|u\|_{W^{2, p}(\mathrm{D})}^{p}$ where the constant $C(p)>0$ depends only on $p$ :

$$
C(p)^{p}=2^{p-1}\left(\frac{3}{2}+\frac{1}{(2-3 / p)^{p}} \frac{1}{8}\left(\frac{3}{4 \pi}\right)^{p}\right) .
$$

For functions $u$ in $W^{2, p}(\Omega)$, the inequality (6.13) is derived directly from the previous one using the triangular inequality.

Lemma 6.9. Given $q$ such that $\frac{1}{p}-\frac{1}{3}<\frac{1}{q}<\frac{1}{3}$ like in (1.6), for any $u \in W^{2, p}(\Omega)$,

$$
\left\|u-\mathbf{p}^{\mathcal{T}} u\right\|_{L^{p}(\Omega)} \leq \frac{|\Omega|^{1 / p-1 / q}}{(1-3 p / q)^{1 / p}} h\|\nabla u\|_{L^{q}(\Omega)} \leq C h\|u\|_{W^{2, p}(\Omega)}
$$

where $C>0$ is constant that depends only on $\Omega$ and $p$.

Proof. Recall that $\mathbf{p}^{\mathcal{T}} u=\left(u\left(x_{\mathrm{K}}\right), u\left(x_{\mathrm{A}}\right), u\left(x_{\mathrm{E}}\right), u\left(x_{\mathrm{F}}\right)\right)_{\mathrm{K} \in \mathcal{M}, \mathrm{A} \in \mathcal{N}, \mathrm{E} \in \mathcal{E}, \mathrm{F} \in \mathcal{F}}$ is a triple of piecewise constant functions, namely $u^{\mathcal{M}}, u^{\mathcal{N}}$ and $u^{\mathcal{F E}}$ and according to the notation (3.3),

$$
\left\|u-\mathbf{p}^{\mathcal{T}} u\right\|_{L^{p}(\Omega)}=\left(\frac{1}{3}\left(\int_{\Omega}\left|u-u^{\mathcal{M}}\right|^{p}+\int_{\Omega}\left|u-u^{\mathcal{N}}\right|^{p}+\int_{\Omega}\left|u-u^{\mathcal{F} \mathcal{E}}\right|^{p}\right)\right)^{1 / p} .
$$

For each of these 3 piecewise constant functions, the error is split into integrals on the 8 tetrahedra that form a diamond, namely the $\left(\mathrm{D}_{i}\right)_{i=1 \ldots 8}$ as defined by eq. (2.1). Hence, it is sufficient to estimate some integrals of the form $\int_{\mathrm{D}_{i}}\left|u(z)-u\left(x_{\mathrm{C}}\right)\right|^{p} d z$ for any $\mathrm{C}=\mathrm{K}, \mathrm{L}, \mathrm{A}, \mathrm{B}, \mathrm{E}, \mathrm{F}$. If $u$ is a function in $C^{2}(\bar{\Omega})$, then $u(z)-u\left(x_{\mathrm{C}}\right)=\int_{0}^{1} \nabla u(t z+$ $\left.(1-t) x_{\mathrm{C}}\right) \cdot\left(z-x_{\mathrm{C}}\right) d t$ and

$$
\begin{aligned}
\int_{\mathrm{D}_{i}}\left|u(z)-u\left(x_{\mathrm{C}}\right)\right|^{p} d z \leq \int_{\mathrm{D}_{i}} \int_{0}^{1}\left|\nabla u\left(t z+(1-t) x_{\mathrm{C}}\right)\right|^{p} h_{\mathrm{D}}^{p} d t d z \\
\quad \leq h_{\mathrm{D}}^{p} \int_{0}^{1} \frac{d t}{t^{3}} \int_{\mathrm{D}_{i}(t)}|\nabla u(x)|^{p} d x \leq h_{\mathrm{D}}^{p} \int_{0}^{1} \frac{d t}{t^{3}}\left(\int_{\mathrm{D}_{i}}|\nabla u(x)|^{q} d x\right)^{p / q}\left|\mathrm{D}_{i}(t)\right|^{1-p / q}
\end{aligned}
$$

where $\mathrm{D}_{i}(t)=\left\{t z+(1-t) x_{\mathrm{C}}, z \in \mathrm{D}_{i}\right\} \subset \mathrm{D}_{i}$ because $\mathrm{D}_{i}$ is convex. Now $\left|\mathrm{D}_{i}(t)\right|=t^{3}\left|\mathrm{D}_{i}\right|$ and then

$$
\begin{aligned}
\int_{\mathrm{D}_{i}}\left|u(z)-u\left(x_{\mathrm{C}}\right)\right|^{p} d z \leq h_{\mathrm{D}}^{p}\left|\mathrm{D}_{i}\right|^{1-p / q}\|\nabla u\|_{L^{q}\left(\mathrm{D}_{i}\right)}^{p} & \int_{0}^{1} t^{-3 p / q} d t \\
& =\frac{1}{1-3 p / q} h_{\mathrm{D}}^{p}\left|\mathrm{D}_{i}\right|^{1-p / q}\|\nabla u\|_{L^{q}\left(\mathrm{D}_{i}\right)}^{p} .
\end{aligned}
$$

Each of the 3 integrals in eq. (6.17) is bounded by $\frac{h^{p}}{1-3 p / q}\|\nabla u\|_{L^{q}(\Omega)}^{p}|\Omega|^{1-p / q}$ so that, using the Sobolev's embedding $W^{2, p}(\Omega) \subset W^{1, q}(\Omega)$, the inequalities (6.16) hold.

6.3. Main result and proof. Our main result is the following.

THEOREM 6.10 (Error estimates). Assume that the flux $\varphi$ satisfies assumptions (1.2), (1.3), (1.4) on the one hand and assumptions (6.5), (6.6) and (6.7) on the other hand. Consider $f \in L^{p^{\prime}}(\Omega)$ and assume that the solution $u$ to (1.1) belongs to $W^{2, p}(\Omega)$. 
For any mesh $\mathcal{T}$ on $\Omega$ there exists a constant $C>0$ depending on the norm $\|u\|_{W^{2, p}}$, the regularity parameter $c_{r}(\mathcal{T})$, the data $\Omega, f, g,\left(b_{i}\right)_{1 \leq i \leq 5}$ and $\left(c_{i}\right)_{1 \leq i \leq 4}$, such that

$\left\|u-u^{\mathcal{M}}\right\|_{L^{p}}+\left\|u-u^{\mathcal{N}}\right\|_{L^{p}}+\left\|u-u^{\mathcal{F E}}\right\|_{L^{p}}+\left\|\nabla u-\nabla_{\delta u}^{\mathcal{T}} u^{\mathcal{T}}\right\|_{L^{p}} \leq C\|u\|_{W^{2, p}(\Omega)} h^{1 /(p-1)}$

where $u^{\mathcal{T}}=\left(u^{\mathcal{M}}, u^{\mathcal{N}}, u^{\mathcal{F E}}\right) \in X$ and $\delta u^{\mathcal{T}}=\mathbf{p}^{\mathcal{T}} g$ (eq. (5.3)) are the solutions to eq. (5.4).

Proof. With the discrete fluxes from definition 6.3, the exact solution verifies the system of equations

$$
-\operatorname{div}_{\mathcal{T}}\left(\psi_{\mathcal{T}}(\nabla u)\right)=\pi^{\mathcal{T}} f
$$

that is the continuous counterpart of the scheme (5.4). Comparing equation (6.19) on the exact solution $u$ and (5.4) on the approximation $u^{\mathcal{T}}$, it is obvious that

$$
-\operatorname{div}_{\mathcal{T}}\left(\psi_{\mathcal{T}}(\nabla u)\right)+\operatorname{div}_{\mathcal{T}}\left(\varphi^{\mathcal{D}}\left(\nabla_{\delta u}^{\mathcal{T}} u^{\mathcal{T}}\right)\right)=0
$$

and equivalently

$$
-\operatorname{div}_{\mathcal{T}}\left(\varphi^{\mathcal{D}}\left(\nabla_{\delta u}^{\mathcal{T}} u^{\mathcal{T}}\right)-\varphi^{\mathcal{D}}\left(\nabla_{\mathbf{p}_{g}}^{\mathcal{T}} \mathbf{p}^{\mathcal{T}} u\right)\right)=-\operatorname{div}_{\mathcal{T}}\left(R_{\mathcal{T}}(u)\right),
$$

where $R_{\mathcal{T}}(u)=\psi_{\mathcal{T}}(\nabla u)-\varphi^{\mathcal{D}}\left(\nabla_{\delta u}^{\mathcal{T}} \mathbf{p}^{\mathcal{T}} u\right) \in \mathbf{Q}$ is given in definition 6.3. Since we take $\delta u^{\mathcal{T}}=\mathbf{p}^{\mathcal{T}} g$, using the discrete duality identity (theorem 4.1 ), the error equation reads

$$
\begin{aligned}
\left(\varphi^{\mathcal{D}}\left(\nabla_{\delta u}^{\mathcal{T}} u^{\mathcal{T}}\right)-\varphi^{\mathcal{D}}\left(\nabla_{\delta u}^{\mathcal{T}} \mathbf{p}^{\mathcal{T}} u\right), \nabla_{\delta u}^{\mathcal{T}} u^{\mathcal{T}}-\nabla_{\delta u}^{\mathcal{T}} \mathbf{p}^{\mathcal{T}} u\right)_{\mathbf{Q}} & \\
& =\left(R_{\mathcal{T}}(u), \nabla_{\delta u}^{\mathcal{T}} u^{\mathcal{T}}-\nabla_{\delta u}^{\mathcal{T}} \mathbf{p}^{\mathcal{T}} u\right)_{\mathbf{Q}}
\end{aligned}
$$

Under assumption (6.5), the error equation (6.20) proves that

$$
c_{3} \int_{\Omega}\left|\nabla_{0}^{\mathcal{T}}\left(u^{\mathcal{T}}-\mathbf{p}^{\mathcal{T}} u\right)\right|^{p} \leq \int_{\Omega} R_{\mathcal{T}}(u) \cdot \nabla_{0}^{\mathcal{T}}\left(u^{\mathcal{T}}-\mathbf{p}^{\mathcal{T}} u\right)
$$

and then, using the inequality of Hölder,

$$
\begin{aligned}
\left(c_{3}\right)^{p^{\prime}} \int_{\Omega}\left|\nabla_{0}^{\mathcal{T}}\left(u^{\mathcal{T}}-\mathbf{p}^{\mathcal{T}} u\right)\right|^{p} \leq \int_{\Omega}\left|R_{\mathcal{T}}(u)\right|^{p^{\prime}} & \\
& \leq\left(\left\|R_{\mathcal{T}}^{1}(u)\right\|_{L^{p^{\prime}}(\Omega)}+\left\|R_{\mathcal{T}}^{2}(u)\right\|_{L^{p^{\prime}}(\Omega)}\right)^{p^{\prime}} .
\end{aligned}
$$

And from lemma 6.6 and 6.7, there exists a constant $C>0$ that depends on $c_{r}(\mathcal{T})$ and on the $\left(b_{i}\right)$, the $\left(c_{i}\right)$ and $p$ such that

$$
\left\|\nabla_{0}^{\mathcal{T}}\left(u^{\mathcal{T}}-\mathbf{p}^{\mathcal{T}} u\right)\right\|_{L^{p}(\Omega)} \leq C\|u\|_{W^{2, p}(\Omega)} h^{1 /(p-1)}
$$

since $p^{\prime} / p=1 /(p-1)$. The proof is easily completed using lemma 6.8 to get the estimate on $\left\|\nabla u-\nabla_{\mathbf{p}_{g}}^{\mathcal{T}} u^{\mathcal{T}}\right\|_{L^{p}(\Omega)}$ and theorem 4.2 and lemma 6.9 to get the estimate on $\left\|u-\mathbf{p}^{\mathcal{T}} u\right\|_{L^{p}(\Omega)}$, because $1 /(p-1) \leq 1$ (so that $h \leq h^{1 /(p-1)}$ for $h \leq 1$ ). 
7. Numerical tests. We exhibit the efficiency of this 3D DDFV scheme on the linear anisotropic problem $\varphi(x, \xi)=\mathbf{K}(x) \xi$. For all the tests, the source term $f$ and the boundary data $g$ are built in such a way that the solution of (1.1) is a given function $u$. The accuracy of the scheme is investigated in the $L^{2}$ and discrete $H^{1}$ norms (from eq. (6.2)). The maximum principle is also examined.

The tests are carried out on four families of meshes as illustrated on figure 7.1: some standard cubic and tetrahedrical meshes (figures 7.1(a) and 7.1(b)), a distorted family of meshes proposed in [7] (figure 7.1(c)) and checkerboard-like meshes (figure $7.1(\mathrm{~d})$ ) that present a great amount of non conformal faces.

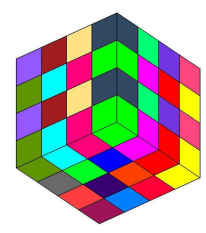

(a) Mesh 1: Cubes.

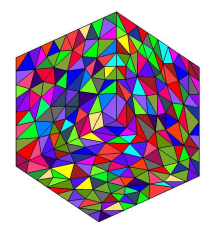

(b) Mesh 2: Tetrahedra.

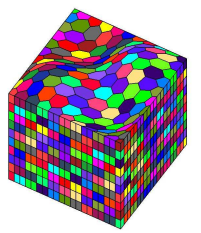

(c) Mesh 3: Prismes.

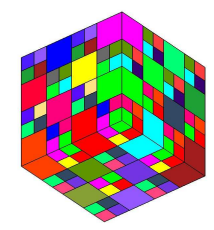

(d) Mesh 4: Checkerboards.

Figure 7.1. The different meshes. Meshes 2 were generated with TetGen [25], meshes 3 are courtesy of K. Lipnikov and Meshes 4 were generated with PELICANS [19].

\begin{tabular}{||r||r|r||r|r||r|r||r|r||}
\hline \multicolumn{1}{||c||}{} & \multicolumn{2}{c||}{ Mesh 1 } & \multicolumn{2}{c||}{ Mesh 2 } & \multicolumn{2}{c||}{ Mesh 3 } & \multicolumn{2}{c||}{ Mesh 4 } \\
\hline$\#$ & Nb cv & Nb un & Nb cv & Nb un & Nb cv & Nb un & Nb cv & Nb un \\
\hline 1 & 8 & 27 & 215 & 737 & 36 & 239 & 1210 & 12179 \\
\hline 2 & 64 & 343 & 2003 & 7777 & 288 & 2543 & 8820 & 96759 \\
\hline 3 & 512 & 3375 & 3898 & 15495 & 2304 & 23135 & 28830 & 325739 \\
\hline 4 & 4096 & 29791 & 7711 & 31139 & 18432 & 196799 & - & - \\
\hline 5 & 32768 & 250047 & 15266 & 62419 & - & - & - & - \\
\hline
\end{tabular}

Number of primary control volumes and number of unknowns.

Test 1: in this first test, the exact solution is quadratic and there is a mild anisotropy. The data are

$$
\mathbf{K}=\left(\begin{array}{ccc}
1 & 0.5 & 0 \\
0.5 & 1 & 0.5 \\
0 & 0.5 & 1
\end{array}\right), \quad u(x, y, z)=16(x(1-x)+y(1-y)+z(1-z))
$$

Test 2: it is the test proposed in [7] where the permeability is strongly heterogeneous and anisotropic and the exact solution is sharp. The data are

$$
\begin{aligned}
& \mathbf{K}(x, y, z)=\left(\begin{array}{ccc}
y^{2}+z^{2}+1 & -x y & -x z \\
-x y & x^{2}+z^{2}+1 & -y z \\
-x z & -y z & x^{2}+y^{2}+1
\end{array}\right), \\
& u(x, y, z)=x^{3} y^{2} z+x \sin (2 \pi x z) \sin (2 \pi x y) \sin (2 \pi z) .
\end{aligned}
$$

Tests 3 and 4: they are built from a rotating and heterogeneous anisotropic 
permeability with a harmonic solution. The data are

$$
\begin{array}{r}
\mathbf{K}=\left(\begin{array}{ccc}
\frac{K_{1} x^{2}+K_{2} y^{2}}{x^{2}+y^{2}} & \frac{\left(K_{1}-K_{2}\right) x y}{x^{2}+y^{2}} & 0 \\
\frac{\left(K_{1}-K_{2}\right) x y}{x^{2}+y^{2}} & \frac{K_{2} x^{2}+K_{1} y^{2}}{x^{2}+y^{2}} & 0 \\
0 & 0 & K_{3} z+1.0
\end{array}\right), \\
\quad u(x, y, z)=\sin (2 \pi x) \sin (2 \pi y) \sin (2 \pi z)
\end{array}
$$

and the values of $K_{1}, K_{2}, K_{3}$ for tests 3 and 4 are given in table 7.2.

\begin{tabular}{||c||c|c|c||}
\hline & $K_{1}$ & $K_{2}$ & $K_{3}$ \\
\hline \hline Test 3 & 1 & 0.1 & 1 \\
\hline Test 4 & 1 & 0.001 & 10 \\
\hline \multicolumn{4}{|c||}{ TABLE 7.2}
\end{tabular}

$K_{1}$ and $K_{2}$ for tests 3 and 4.

We observe in tables 7.3-7.6 an order of convergence closed to 2 in $L^{2}$-norm and order 1 convergence in the discrete $H^{1}$ norm. This super-convergence is classically observed for finite volume schemes, but it remains an open problem for general meshes, even for 2D-DDFV.

As the exact solution lies for test 1 in $[0,12]$ and for test $2,3,4$ in $[-1,1]$, we observe in table 7.7 that the positivity of the scheme is fulfilled, and that the maximum principle is only achieved asymptotically. In the highly anisotropic and heterogeneous tests 3 and 4, we observe in table 7.8 that neither the positivity, neither the maximum principle hold, as it was already the case for the 2D DDFV schemes and for all the linear schemes (see $[15,5])$. Only nonlinear schemes can ensure the maximum principle in such severe situations $([21,24,20,13])$.

\begin{tabular}{||c||cc|cc||cc|cc||}
\hline \multicolumn{1}{||c||}{} & \multicolumn{4}{c||}{ Mesh 1} & \multicolumn{4}{c||}{ Mesh 4} \\
\hline \hline$\#$ & $\|\cdot\|_{2}$ & Rate & $\|\cdot\|_{H_{1}}$ & Rate & $\|\cdot\|_{2}$ & Rate & $\|\cdot\|_{H_{1}}$ & Rate \\
\hline 1 & $0.18 \mathrm{e}+00$ & - & $0.12 \mathrm{e}+01$ & - & $0.78 \mathrm{e}+00$ & - & $0.28 \mathrm{e}+01$ & - \\
\hline 2 & $0.37 \mathrm{e}-01$ & 2.02 & $0.49 \mathrm{e}+00$ & 1.10 & $0.22 \mathrm{e}+00$ & 1.62 & $0.15 \mathrm{e}+01$ & 0.80 \\
\hline 3 & $0.24 \mathrm{e}-01$ & 1.91 & $0.39 \mathrm{e}+00$ & 0.96 & $0.57 \mathrm{e}-01$ & 1.82 & $0.77 \mathrm{e}+00$ & 0.90 \\
\hline 4 & $0.15 \mathrm{e}-01$ & 2.10 & $0.30 \mathrm{e}+00$ & 1.09 & $0.14 \mathrm{e}-01$ & 1.91 & $0.39 \mathrm{e}+00$ & 0.95 \\
\hline 5 & $0.95 \mathrm{e}-02$ & 1.87 & $0.25 \mathrm{e}+00$ & 0.93 & - & - & - & - \\
\hline
\end{tabular}

Relative $L^{2}$ and $H^{1}$ errors and rate of convergence for test 1.

\section{REFERENCES}

[1] R. A. Adams. Sobolev spaces. Academic press, 1970.

[2] B. Andreianov, M. Bendahmane, and K. Karlsen. A gradient reconstruction formula for finitevolume schemes and discrete duality. In Finite Volume For Complex Applications, Problems And Perspectives. 5th International Conference. London (UK) Wiley, 2008.

[3] B. Andreianov, F. Boyer, and F. Hubert. Discrete duality finite volume schemes for Leray-Lions type elliptic problems on general 2D-meshes. Numerical method in PDE, 23(1):145-195, 2007.

[4] B. Andreianov, M. Gutnic, and P. Wittbold. Convergence of finite volume approximations for a nonlinear elliptic-parabolic problem : a "continuous approach". SIAM Journal on Numerical Analysis, 42(1):228-251, 2004. 


\begin{tabular}{||c||cc|cc||cc|cc||}
\hline \multicolumn{1}{||c||}{} & \multicolumn{4}{c||}{ Mesh 1 } & \multicolumn{4}{c||}{ Mesh 3 } \\
\hline \hline$\#$ & $\|\cdot\|_{2}$ & Rate & $\|\cdot\|_{H_{1}}$ & Rate & $\|\cdot\|_{2}$ & Rate & $\|\cdot\|_{H_{1}}$ & Rate \\
\hline 1 & $0.25 \mathrm{e}+00$ & - & $0.85 \mathrm{e}+00$ & - & $0.12 \mathrm{e}-01$ & - & $0.18 \mathrm{e}+00$ & - \\
\hline 2 & $0.54 \mathrm{e}-01$ & 1.79 & $0.39 \mathrm{e}+00$ & 0.92 & $0.33 \mathrm{e}-02$ & 1.85 & $0.89 \mathrm{e}-01$ & 1.03 \\
\hline 3 & $0.17 \mathrm{e}-01$ & 1.56 & $0.20 \mathrm{e}+00$ & 0.87 & $0.15 \mathrm{e}-02$ & 1.92 & $0.58 \mathrm{e}-01$ & 1.05 \\
\hline 4 & $0.44 \mathrm{e}-02$ & 1.82 & $0.97 \mathrm{e}-01$ & 1.01 & - & - & - & - \\
\hline 5 & $0.11 \mathrm{e}-02$ & 1.92 & $0.47 \mathrm{e}-01$ & 1.02 & - & - & - & - \\
\hline
\end{tabular}

Relative $L^{2}$ and $H^{1}$ errors and rate of convergence for test 2.

\begin{tabular}{||c||cc|cc||cc|cc||}
\hline \multicolumn{1}{||c||}{} & \multicolumn{4}{c||}{ Mesh 1 } & \multicolumn{4}{c||}{ Mesh 2} \\
\hline \hline$\#$ & $\|\cdot\|_{2}$ & Rate & $\|\cdot\|_{H_{1}}$ & Rate & $\|\cdot\|_{2}$ & Rate & $\|\cdot\|_{H_{1}}$ & Rate \\
\hline 1 & $0.34 \mathrm{e}+00$ & - & $0.25 \mathrm{e}+00$ & - & $0.20 \mathrm{e}+01$ & - & $0.12 \mathrm{e}+01$ & - \\
\hline 2 & $0.56 \mathrm{e}-01$ & 2.14 & $0.62 \mathrm{e}+00$ & 0.95 & $0.39 \mathrm{e}-01$ & 2.06 & $0.51 \mathrm{e}+00$ & 1.11 \\
\hline 3 & $0.16 \mathrm{e}-01$ & 1.67 & $0.36 \mathrm{e}+00$ & 0.72 & $0.25 \mathrm{e}-01$ & 2.00 & $0.40 \mathrm{e}+00$ & 1.11 \\
\hline 4 & $0.41 \mathrm{e}-02$ & 1.86 & $0.18 \mathrm{e}+00$ & 0.91 & $0.16 \mathrm{e}-01$ & 1.97 & $0.31 \mathrm{e}+00$ & 1.09 \\
\hline 5 & $0.10 \mathrm{e}-02$ & 1.96 & $0.93 \mathrm{e}-01$ & 0.97 & $0.98 \mathrm{e}-02$ & 2.00 & $0.24 \mathrm{e}+00$ & 1.04 \\
\hline
\end{tabular}

Relative $L^{2}$ and $H^{1}$ errors and rate of convergence for test 3.

[5] F. Boyer and F. Hubert. Benchmark on anisotropic problems, the ddfv discrete duality finite volumes and m-ddfv schemes. In R. Eymard and J.-M. Hérard, editors, Finite Volumes for Complex Applications $V$, pages 735-750. Wiley, 2008.

[6] S. C. Brenner and L. R. Scott. The Mathematical Theory of Finite Element Methods, volume 15 of Texts in Applied Mathematics. Springer New York, 2008.

[7] F. Brezzi, A. Buffa, and K. Lipnikov. Mimetic finite differences for elliptic problems. ESAIM: Mathematical Modeling and Numerical Analysis, 43:277-295, 2009.

[8] Y. Coudière, C. Pierre, and R. Turpault. A 2d/3d discrete duality finite volume scheme. application to ecg simulation. International Journal on Finite Volumes, 6(1), 2009. http://hal.archives-ouvertes.fr/hal-00328251/fr.

[9] Y. Coudière, J.-P. Vila, and P. Villedieu. Convergence rate of a finite volume scheme for a two dimensional convection-diffusion problem. M2AN, 33(3):493-516, 1999.

[10] K. Domelevo and P. Omnes. A finite volume method for the Laplace equation on almost arbitrary two-dimensional grids. M2AN, 39(6):1203-1249, 2005.

[11] J. Droniou. Error estimates for the convergence of a finite volume discretization of convectiondiffusion equations. Numer. Math., 11(1):1-32, 2003.

[12] J. Droniou and R. Eymard. A mixed finite volume scheme for anisotropic diffusion problems on any grid. Num. Math., 105(1):35-71, 2006.

[13] J. Droniou and C. Le Potier. Construction and convergence study of local-maximum-principle preserving schemes for elliptic equations. submitted in 2009.

[14] R. Eymard, T. Gallouët, and R. Herbin. Handbook of Numerical Analysis, Vol. VII, chapter Finite Volume Methods. North-Holland, 2000.

[15] R. Herbin and F. Hubert. Benchmark on discretization schemes for anisotropic diffusion problems on general grids for anisotropic heterogeneous diffusion problems. In R. Eymard and J.-M. Hérard, editors, Finite Volumes for Complex Applications V, pages 659-692. Wiley, 2008.

[16] F. Hermeline. A finite volume method for the approximation of diffusion operators on distorted meshes. Journal of computational Physics, 160:481-499, 2000.

[17] F. Hermeline. Approximation of 2-d and 3-d diffusion operators with variable full tensor coefficients on arbitrary meshes. Comput. Methods Appl. Mech. Engrg., 196, 2007.

[18] F. Hermeline. A finite volume method for approximating 3d diffusion operators on general meshes. Journal of computational Physics, 228(16):5763-5786, 2009.

[19] IRSN, https://gforge.irsn.fr/gf/project/pelicans/. PELICANS.

[20] C. Le Potier. Schémas volumes finis pour des opérateurs de diffusion fortement anisotropes sur 


\begin{tabular}{||c||cc|cc||cc|cc||}
\hline \multicolumn{1}{||c||}{} & \multicolumn{5}{c||}{ Mesh 1} & \multicolumn{4}{c||}{ Mesh 2} \\
\hline \hline$\#$ & $\|\cdot\|_{2}$ & Rate & $\|\cdot\|_{H_{1}}$ & Rate & $\|\cdot\|_{2}$ & Rate & $\|\cdot\|_{H_{1}}$ & Rate \\
\hline 1 & $0.42 \mathrm{e}+00$ & - & $0.19 \mathrm{e}+01$ & - & $0.25 \mathrm{e}+00$ & - & $0.17 \mathrm{e}+01$ & - \\
\hline 2 & $0.25 \mathrm{e}+00$ & 0.61 & $0.29 \mathrm{e}+01$ & -0.49 & $0.49 \mathrm{e}-01$ & 0.83 & $0.79 \mathrm{e}+00$ & 1.01 \\
\hline 3 & $0.66 \mathrm{e}-01$ & 1.74 & $0.16 \mathrm{e}+01$ & 0.82 & $0.32 \mathrm{e}-01$ & 1.90 & $0.61 \mathrm{e}+00$ & 1.09 \\
\hline 4 & $0.17 \mathrm{e}-01$ & 1.88 & $0.80 \mathrm{e}+00$ & 0.93 & $0.20 \mathrm{e}-01$ & 1.94 & $0.49 \mathrm{e}+00$ & 0.94 \\
\hline 5 & $0.43 \mathrm{e}-02$ & 1.94 & $0.40 \mathrm{e}+00$ & 0.97 & $0.13 \mathrm{e}-01$ & 2.05 & $0.38 \mathrm{e}+00$ & 1.06 \\
\hline
\end{tabular}

Relative $L^{2}$ and $H^{1}$ errors and rate of convergence for test 4 .

\begin{tabular}{|c|c|c|c|c|c|c|c|c|c|}
\hline & \multicolumn{4}{|c|}{ Test 1} & & \multicolumn{4}{|c|}{ Test 2} \\
\hline & \multicolumn{2}{|c|}{ Mesh 1} & \multicolumn{2}{|c|}{ Mesh 4} & & \multicolumn{2}{|c|}{ Mesh 1} & \multicolumn{2}{|c|}{ Mesh 3} \\
\hline \# & $u_{\min }$ & $u_{\max }$ & $u_{\min }$ & $u_{\max }$ & $\#$ & $u_{\min }$ & $u_{\max }$ & $u_{\min }$ & $u_{\max }$ \\
\hline 1 & 0.000 & 11.938 & 0.000 & 12.023 & 1 & -0.859 & 1.047 & -0.859 & 1.047 \\
\hline 2 & 0.000 & 12.022 & 0.000 & 11.999 & $\overline{2}$ & -0.930 & 1.047 & -0.861 & 1.049 \\
\hline 3 & 0.000 & 11.992 & 0.000 & 12.000 & 3 & -0.859 & 1.047 & -0.861 & 1.049 \\
\hline 4 & 0.000 & 12.006 & 0.000 & 12.000 & 4 & -0.859 & 1.047 & - & - \\
\hline 5 & 0.000 & 12.004 & - & - & 5 & -0.860 & 1.048 & - & - \\
\hline
\end{tabular}

Maximum principle for tests 1 and 2.

\begin{tabular}{|c|c|c|c|c|c|c|c|c|c|}
\hline & \multicolumn{4}{|c|}{ Test 3} & & \multicolumn{4}{|c|}{ Test 4} \\
\hline & \multicolumn{2}{|c|}{ Mesh 1} & \multicolumn{2}{|c|}{ Mesh 2} & & \multicolumn{2}{|c|}{ Mesh 1} & \multicolumn{2}{|c|}{ Mesh 2} \\
\hline \# & $u_{\min }$ & $u_{\max }$ & $u_{\min }$ & $u_{\max }$ & $\#$ & $u_{\min }$ & $u_{\max }$ & $u_{\min }$ & $u_{\max }$ \\
\hline 1 & -0.663 & 0.682 & -1.072 & 1.178 & 1 & -0.792 & 0.892 & -1.511 & 1.305 \\
\hline 2 & -0.977 & 0.985 & -1.016 & 1.086 & 2 & -2.174 & 2.105 & -1.032 & 1.131 \\
\hline 3 & -0.999 & 1.001 & -1.012 & 1.028 & 3 & -1.343 & 1.336 & -1.067 & 1.047 \\
\hline 4 & -1.000 & 1.000 & -1.003 & 1.021 & 4 & -1.083 & 1.083 & -1.021 & 1.030 \\
\hline 5 & -1.000 & 1.000 & -1.015 & 1.015 & 5 & -1.020 & 1.020 & -1.051 & 1.020 \\
\hline
\end{tabular}

Maximum principle for tests 3 and 4.

des maillages non structurés. C. R. Math. Acad. Sci. Paris, 340(12):921-926, 2005.

[21] C. Le Potier. Benchmark for anisotropic problems. numerical results with two cell-centered finite volume schemes for heteregeneous anisotropic diffusion operators. In R. Eymard and J.-M. Hérard, editors, Finite Volumes for Complex Applications V, pages 735-750. Wiley, 2008.

[22] J. L. Leray, J.; Lions. Quelques résultats de visik sur les problèmes elliptiques non linéaires par les méthodes de minty-browder. Séminaire Jean Leray, 1:1-19, 1964.

[23] C. Pierre. Modélisation et simulation de l'activité électrique du coeur dans le thorax, analyse numérique et méthodes de volumes finis. PhD thesis, Université de Nantes, 2005.

[24] D. Svyatskiy. Benchmark for anisotropic problems. nonlinear monotone finite volume method. In R. Eymard and J.-M. Hérard, editors, Finite Volumes for Complex Applications V, pages 735-750. Wiley, 2008.

[25] WIAS, http://tetgen.berlios.de. TETGEN. 